\title{
REVIEW
}

Open Access

\section{Multisector nutrition gains amidst evidence scarcity: scoping review of policies, data and interventions to reduce child stunting in Afghanistan}

Christine Kim ${ }^{1 *}$ (D) Ghulam Farooq Mansoor ${ }^{2}$, Pir Mohammad Paya ${ }^{2}$, Mohammad Homayoun Ludin ${ }^{3}$, Mohammad Javed Ahrar ${ }^{4}$, Mohammad Omar Mashal ${ }^{2}$ and Catherine S. Todd ${ }^{5}$

\begin{abstract}
Background: Child health indicators have substantially improved across the last decade, yet Afghanistan has among the highest child stunting and malnutrition rates in Asia. Multisectoral approaches were recently introduced but evidence for this approach to improve support for and implementation of child nutrition programmes is limited compared to other countries.

Methods: We reviewed policy and programme data to identify best practices and gaps surrounding child malnutrition in Afghanistan. We conducted a scoping review using broad search categories and approaches, including database and website searches, reference hand-searches, purposive policy and programme document request, and key informant interviews. Inclusion and exclusion criteria were developed iteratively, with abstracts and documents assessed against the final criteria. We abstracted documents systematically and summarised and synthesised content to generate the main findings.

Results: We included 18 policies and strategies, 45 data sources and reports, and 20 intervention evaluations. Movement towards multisectoral efforts to address malnutrition at the policy level has started; however, integrated nutrition-specific and nutrition-sensitive interventions are not yet uniformly delivered at the community level. Many data sources capturing nutrition, food security and WASH (water, sanitation and hygiene) indicators are available but indicator definitions are not standardised and there are few longitudinal nutrition surveys. Political will to improve household nutrition status has shown increased government and donor investments in nutrition-sensitive and nutrition-specific programmes through combined small- and large-scale interventions between 2004 and 2013; however, evidence for interventions that effectively decrease stunting prevalence is limited.

(Continued on next page)
\end{abstract}

\footnotetext{
* Correspondence: christine.kim@unc.edu

'Department of Health Policy and Management, Gillings School of Global

Public Health, University of North Carolina at Chapel Hill, Chapel Hill, NC, United States of America

Full list of author information is available at the end of the article
}

(C) The Author(s). 2020 Open Access This article is licensed under a Creative Commons Attribution 4.0 International License, which permits use, sharing, adaptation, distribution and reproduction in any medium or format, as long as you give appropriate credit to the original author(s) and the source, provide a link to the Creative Commons licence, and indicate if changes were made. The images or other third party material in this article are included in the article's Creative Commons licence, unless indicated otherwise in a credit line to the material. If material is not included in the article's Creative Commons licence and your intended use is not permitted by statutory regulation or exceeds the permitted use, you will need to obtain permission directly from the copyright holder. To view a copy of this licence, visit http://creativecommons.org/licenses/by/4.0/. The Creative Commons Public Domain Dedication waiver (http://creativecommons.org/publicdomain/zero/1.0/) applies to the data made available in this article, unless otherwise stated in a credit line to the data. 
(Continued from previous page)

Conclusions: This review shows a breadth of nutrition programme, policy and data in Afghanistan. Multisector approaches faced challenges of reaching sufficient coverage as they often included a package of food security, livelihoods and health interventions but were each implemented independently. Further implementation evidence is needed to aid policy and programmes on effective integration of nutrition, food security and WASH in Afghanistan.

Keywords: child nutrition, Afghanistan, water, sanitation and hygiene, nutrition policy, stunting, micronutrients, fragile and conflict-affected, multisectoral approach

\section{Background}

Globally, malnutrition affects one in three people and is a primary driver of the global burden of disease with high associated economic costs [1]. Africa and Asia lose $11 \%$ of their gross domestic product annually to malnutrition and fragile health systems are further strained by poor child health and development, micronutrient deficiencies, and nutrition-related infectious and noncommunicable diseases $[1,2]$. International partners and countries have increasingly recognised the global challenge of malnutrition and commitments to collaboratively end malnutrition in all forms have coalesced. In 2013, the Lancet published its second series on maternal and child malnutrition, highlighting the lack of highquality evidence for and multisectoral inclusion of nutrition interventions as well as a lack of incorporation of nutrition-sensitive approaches such as water, sanitation and hygiene (WASH), agriculture, and food security into existing nutrition-specific programming [3]. The second Sustainable Development Goal (SDG) aims to "end hunger, achieve food security and improved nutrition, and promote sustainable agriculture" and 12 of 17 SDG indicators address some aspect of nutrition [4]. Thus, investments in nutrition are important to achieving the SDGs.

Malnutrition, specifically including fetal growth restriction, child stunting and wasting, and deficiencies of vitamin $\mathrm{A}$ and zinc, along with suboptimal breastfeeding, causes 3.1 million child deaths or almost $50 \%$ of all deaths under age 5 years annually [5]. The prevalence of malnutrition is significantly higher among fragile and conflict-affected settings [6]. Almost all countries ranked within the highest $10 \%$ of the Global Hunger Index [7] and with the highest stunting rates are classified as fragile or conflict affected [8]. Conflict and fragility affect nutritional status through different pathways and exacerbate poor health outcomes [9, 10]. Nearly $40 \%$ of the world's stunted children live in South Asia [11]. Malnutrition is an indicator of social and political instability as it represents a multifaceted problem linked to poverty, food insecurity, and poor hygiene and health.

However, there is a growing evidence base of effective high impact interventions to reduce preventable malnutrition during the critical developmental window between pregnancy to 24 months of age [12]. These interventions include nutrition-sensitive components, such as WASH programmes, with better associated outcomes than nutrition-specific interventions. Poor sanitation, water quality and hygiene practices increase the risk for diarrheal disease and prevent adequate nutrient absorption in children. Diarrheal diseases are the second leading cause of death in children under 5 years of age, accounting for one in nine child deaths globally $[13,14]$. Most (88\%) diarrhoea-associated deaths are attributable to unsafe water, inadequate sanitation and insufficient hygiene [15]. Hand washing counselling can lead to a $30 \%$ reduction in diarrhoea [16]. Since 2014, the inter-relatedness of sanitation and nutrition has been elevated to a global policy priority. Existing evidence supports at least three direct pathways linking poor WASH access to a child's nutritional status: diarrheal disease, intestinal parasite infections and environmental enteropathy [17]. Although the results of the Sanitation Hygiene Infant Nutrition Efficacy (SHINE) Trial and WASH Benefits Trial found that household-level WASH interventions were unlikely to reduce stunting or diarrhoea, they did not focus on community-wide coverage to achieve an open defecationfree status $[18,19]$. Open defecation continues to be a challenge in Afghanistan and remains a community-level pathway to faecal contaminant exposure [20].

Afghanistan is the only country in the South Asia region in a conflict-affected and fragile situation [8, 21]. Afghanistan also ranks eighth on the Global Hunger Index, 14th for stunting rate and 42nd of 45 countries by the Hunger and Nutrition Commitment Index on country government's political commitment to tackling hunger and malnutrition [1, 7, 22]. Globally, while investments have been made in implementing nutrition programmes and generating robust evidence, peer-reviewed data are limited and programme knowledge, often within the grey literature, is not widely shared or transferred. A 2018 series on nutrition in South Asia included two reviews, one on maternal nutrition interventions and another on optimal breastfeeding interventions. No studies from Afghanistan were eligible for inclusion based on the criteria for these reviews [23, 24]. 
However, there is a need to understand the available nutrition data from Afghanistan to improve knowledge sharing, evidence generation, policy development and programme implementation in the country and in other fragile settings facing similar challenges.

While other scoping reviews focus on evidencebased nutrition interventions, nutrition-related data sources and policies contextualise evidence generation and prioritisation. This scoping review therefore maps nutrition-related policies, data and interventions aiming to reduce child stunting in Afghanistan and the extent to which multisectoral involvement contributed to their success. The objectives were to (1) delineate nutrition policy and programmes and the degree of multisectoral inclusion; (2) describe the scope of available published and grey literature; and (3) identify programme and knowledge gaps. We explore nutrition-related policy, data and evidence sources to make summary recommendations to inform multisectoral efforts to reduce child malnutrition in Afghanistan.

\section{Methods}

\section{Study design}

We conducted a scoping review using the six-stage methodological framework proposed by Arksey and O'Malley [25] and enhanced by Levac et al. [26]. The stages include (1) identifying the research question, (2) searching for relevant sources, (3) selecting sources, (4) extracting data, (5) collating, summarising and reporting, and (6) consulting stakeholders [25, 27]. We selected a scoping exercise rather than a systematic review to map the wide range of published and grey literature, policy and data sources on child stunting in Afghanistan and to better define the gaps within existing practice and research. Additionally, we applied a two-step process to iteratively identify and select sources as well as to engage stakeholders [26]. The first step comprised a rapid desk review with a broad literature review and stakeholder interviews conducted in August 2017 to understand available sources of and gaps in information. A second systematic literature search and expanded hand-search of relevant content was conducted between September 2018 and February 2019. We followed the PRISMA checklist for scoping reviews. No scoping review protocol was registered publicly for this study.

\section{Search strategy}

The initial literature search was conducted through MEDLINE/PubMed and Google Scholar, with the following search terms: (("nutrition sensitive" OR "nutrition specific" OR "WASH" OR "water sanitation hygiene" OR "food security" OR "nutrition" OR "infant and young child feeding" OR "IYCF" OR "maternal nutrition")
AND "Afghanistan"). Websites for Afghan government ministries, non-government organisation (NGO) implementing partners in the health sector and donors were purposively hand-searched for relevant reports. Key informants in the government (Ministry of Public Health $(\mathrm{MoPH})$, Ministry of Rural Rehabilitation and Development, and the Ministry of Agriculture, Irrigation and Livestock (MAIL)), NGOs, members of nutrition-related technical working groups, and provincial health officials were interviewed in person or contacted with an emailbased electronic survey. Stakeholders were engaged initially to understand their past and current nutrition, food security and/or WASH programming as well as the data sources and evidence used to inform their policies and programmes.

A second literature search was conducted across 13 databases, namely MEDLINE/PubMed, Popline, Embase, Global Health, Academic Search Premier, CINAHL Plus with Full Text, EconLit, Education Full Text (H.W. Wilson), Environment Complete, ERIC, GreenFILE, Middle Eastern and Central Asian Studies, and Web of Science. Example search terms included (from PubMed): ("child nutrition" OR "infant and young child feeding" OR child nutrition sciences [Mesh] OR "infant and young child feeding" OR Prenatal Nutritional Physiological Phenomena [Mesh] OR child nutritional physiological phenomena [Mesh] OR ((nutritional status [Mesh] OR "nutrition sensitive" OR "nutrition" OR nutritional requirements [Mesh] OR "nutrition specific" OR "undernutrition" OR "malnutrition" OR hunger OR nutrition assessment [Mesh] OR nutrition surveys [Mesh] OR nutrition policy [Mesh] OR "nutrition during pregnancy" OR "maternal nutrition" OR "maternal undernutrition" OR "maternal malnutrition" OR "Maternal Nutritional Physiological Phenomena" [Mesh] OR "maternal iron supplementation" OR "dietary supplements during pregnancy" OR Food Labeling [Mesh] OR "exclusive breastfeeding" OR "feeding practice" OR dietary diversity OR MUAC OR "mid-upper arm circumference" OR stunting OR wasting) AND (child OR children OR infant)) AND Afghanistan [Mesh]). Table S1 contains the complete search strategy for each database. Reference lists were hand-searched for relevant sources from 14 review papers identified through database searches. A purposive hand-search was then conducted for grey sources from websites of government ministries, NGO implementing partners in the health sector and donors.

\section{Source selection}

Prior to selecting sources, we agreed on broad inclusion and exclusion criteria and used these at the first search 
stage. We then refined these criteria in an iterative process during the first phase of abstract review, which delineated relevant content and sources [26]. This approach helped alleviate uncertainties surrounding source selection, given the broad research question and goal of including grey literature sources.

To map the current child nutrition situation in Afghanistan, we categorised our sources into published articles, grey literature reports, data sources and policy documents. Final inclusion and exclusion criteria are shown in Box 1.

\section{Box 1 Inclusion and exclusion criteria}

Inclusion criteria

Peer-reviewed article

- Peer-reviewed research on nutrition, food security and/or WASH interventions

- Includes a measure of at least one child nutrition outcome or nutrition-related knowledge or behaviour (includes hygiene)

- Specific to Afghanistan

Grey literature

- Programmatic research or evaluation done by third party

- Explicit interventions/programmes on nutrition, food, security and/or WASH with expected changes in nutritional status

- Implemented in Afghanistan

Data source

- Measurement of nutrition-related knowledge, behaviours or outcomes

- Data collected from Afghanistan

Policy document

- All relevant government policies included

Exclusion criteria

- Not publicly disseminated or unavailable to public upon request from source

- Not specific to Afghanistan

- Target population is Afghan refugees no longer in the country or children older than 5 years

- Not written in English

- Global or multi-country study without specific data from Afghanistan, rather only aggregated global or regional estimates

- Agriculture, food security, economic development, or water and sanitation-related programming and/or research without explicit child nutrition component or measures
All relevant data sources and policy documents were included for analysis. Only published articles and grey literature underwent full text screening. Two independent reviewers screened all abstracts/titles (CK and CT) and selected full texts (GFM and MOM). Any disagreements on source inclusion were resolved by the two reviewers and, when necessary, by a third reviewer. We did not appraise source quality as we aimed to include all relevant sources to provide a full landscape of national nutrition programming.

\section{Citation management and data extraction}

All citations were imported into Endnote X7 (Clarivate Analytics, PA, United States of America). Duplicate citations were removed manually during screening. Data were extracted into a Microsoft Excel spreadsheet. Descriptive information from each source was extracted by specified categories. Table 1 lists the data elements extracted for each type of source.

\section{Analysis}

We first collated and summarised the scope of available sources and then described the nutrition programmes that had been evaluated and the extent to which they contained multisectoral approaches. We synthesised information identifying changes to priority areas and outcomes over time in data sources and policy documents, respectively, with iterative identification of programme

Table 1 Data extraction elements from sources

\begin{tabular}{ll}
\hline Types of source & Extraction categories \\
\hline Published peer-reviewed studies & Author(s), publication year, title, \\
and grey literature reports & source type (published or grey), \\
& product of database search or \\
hand search, objectives, study & design (research, evaluation, case \\
& study), location of study (national, \\
& sub-national), population \\
& (households, children $<5$ years, \\
& children 0-24 months), sample size \\
& if applicable, nutrition-related \\
& outcomes measured, multi-sectoral \\
& approach if applicable, enabling \\
& environment, and lessons learned \\
& Citation, description, year of \\
& publication, primary or secondary \\
analysis, sample size, geographic & coverage, child nutrition-related \\
measures, level of summary of & evidence for decision-making and \\
biases in sampling/results \\
interpretation \\
Citation, year, government \\
agency, department, multisectoral \\
approaches, child nutrition-related \\
objectives, main child nutrition- \\
related strategies, changes from last \\
iteration and programmatic responses
\end{tabular}


and knowledge gaps. Only the key findings are presented in this paper.

\section{Results}

\section{Scope of sources}

After removing duplicates from a combination of database, prior desk review and a second hand-search results, 342 source documents were screened by abstract and title. All relevant data reports and policy documents were included, while full-text screening was conducted for 64 peer-reviewed articles and 46 grey literature documents. We classified 25 studies as data reports because they included secondary data analyses of nutrition outcomes and formative research or provided other relevant nutritionrelated evidence but did not include an intervention. Overall, we found 46 data reports, 18 policy documents and 20 full text studies (peer-reviewed articles and grey literature programme reports) that met the inclusion criteria for this scoping review (Fig. 1).

We first provide an overview of government policy responses to child malnutrition. Next, we present the ways in which nutrition status and nutrition-related indicators have been measured and studied in Afghanistan. Finally, we present nutrition-specific and nutrition-sensitive interventions that aimed to improve the nutrition status of children.

\section{Policy responses to child malnutrition}

Multiple policies and strategies have been developed over the past 15 years at the level of government ministries to address nutrition, WASH and food security. More recently, government-wide, multisectoral policies have been introduced to coordinate and empower ministries to collaboratively work towards national nutrition and food security goals. Figure 2 illustrates the timeline for policies and data/evidence published around nutrition, food security and WASH included in this review. We identified 18 relevant policies and strategies that incorporated the nutrition objectives between 2003 and 2018. The first Public Nutrition Policy and Strategy was developed in 2003, with follow-up documents in 2009 and 2015. These policy and strategy documents largely continue the same objectives, with more specific strategies described in the latter two periods such as targeting specific vulnerable populations like pregnant women, adolescent girls and children under 5 years of age. Addressing malnutrition has consistently been a key objective of the health sector, present in both the National Health Policy and National Health Strategy since 2005. In 2009, two specific strategies were developed to address poor infant and young child feeding (IYCF) practices and prevent and reduce micronutrient deficiencies. While these health sector policies and strategies acknowledged multisectoral pathways of malnutrition and the need for coordination between line ministries for effective nutritionsensitive intervention programming, engagement of non-health sector ministries in nutrition-related actions did not occur until after 2010. This is possibly

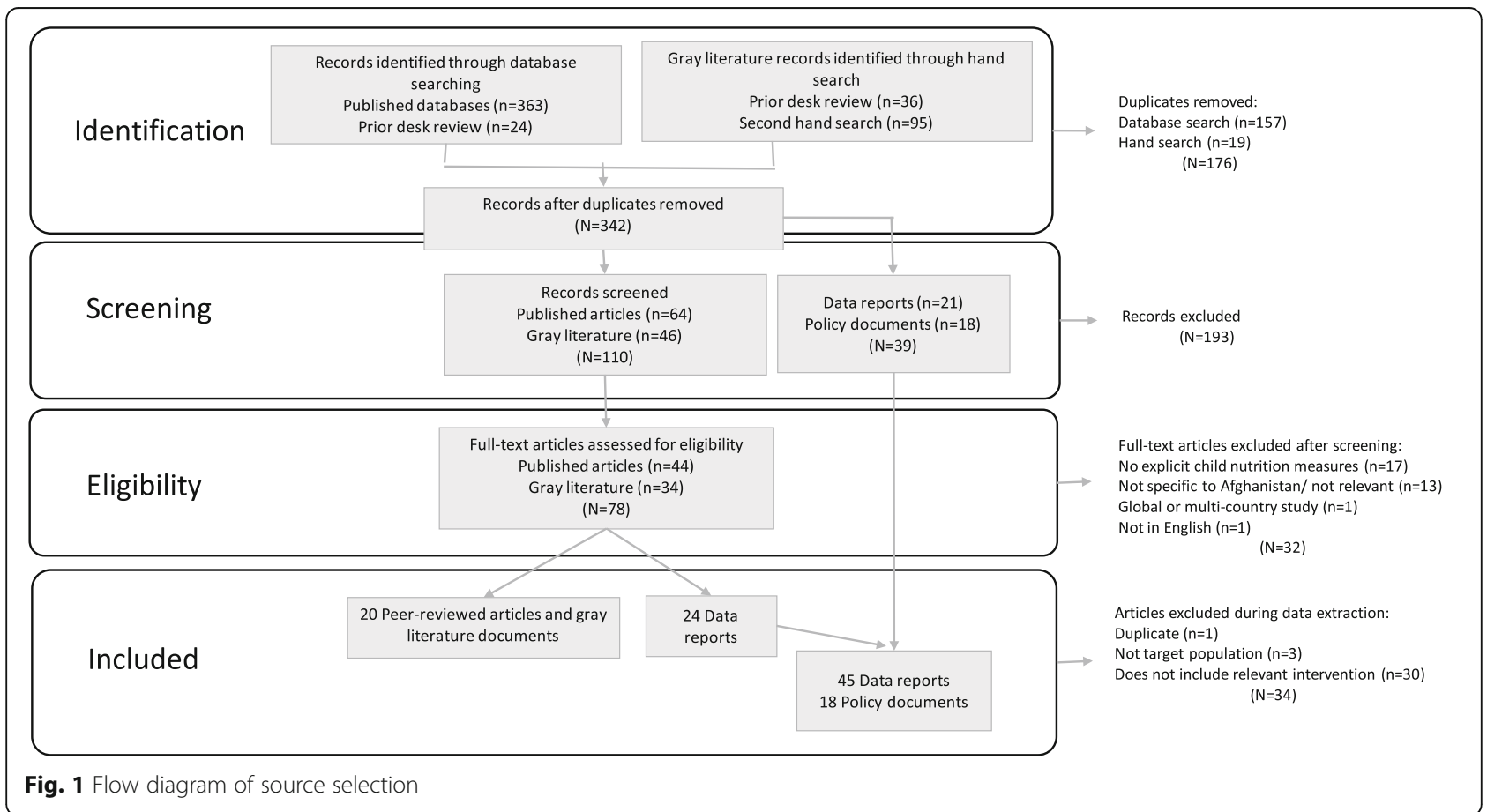




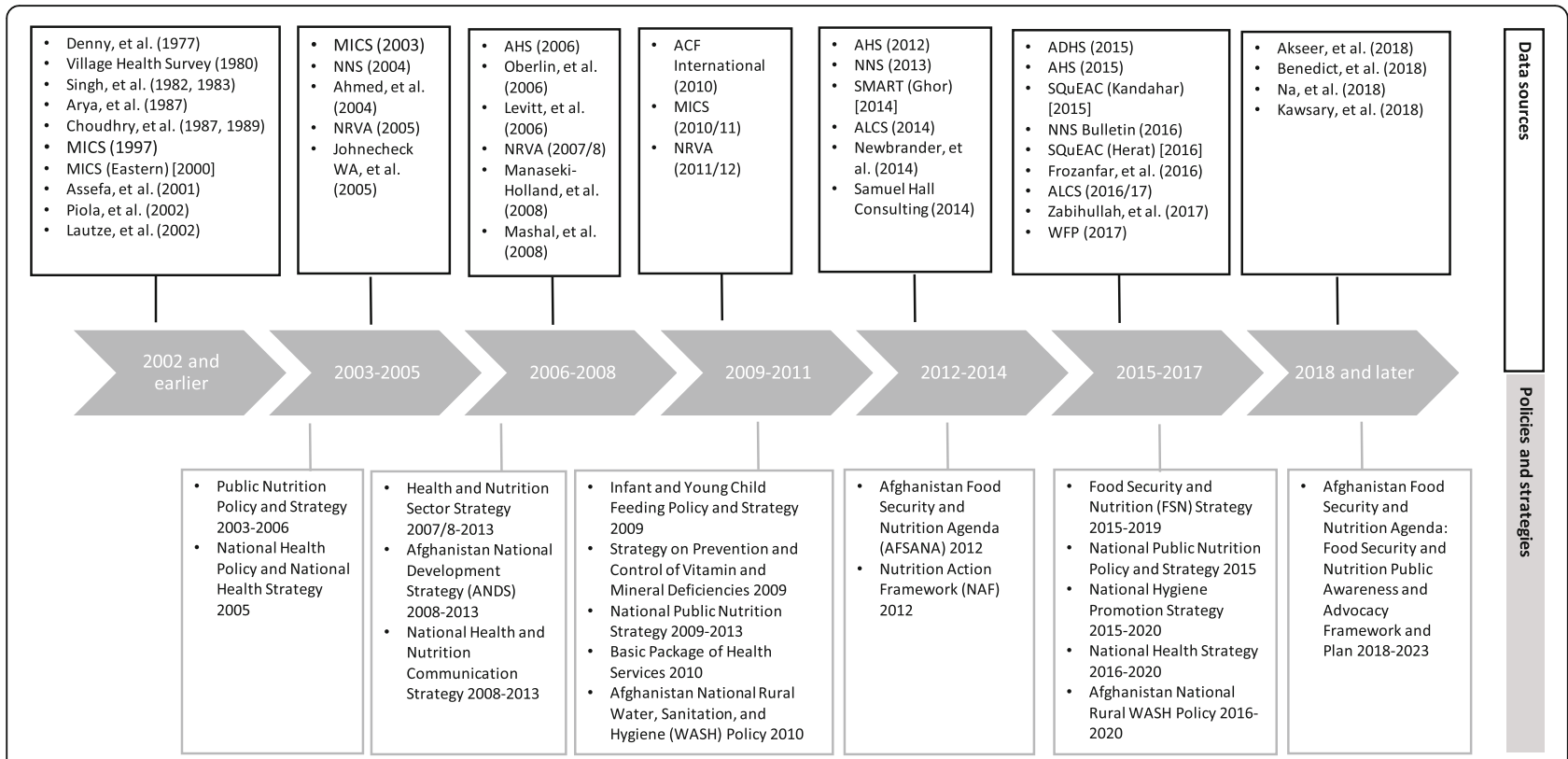

Fig. 2 Timeline of data sources and policies

due to the influence of the Afghanistan National Development Strategy (ANDS) in 2008, which was the country's overall strategy for security, governance, economic growth, social development and poverty reduction to reach the Millennium Development Goals. The ANDS outlined the country's development policies and was the first extensive multisector strategy endorsed by the government and international partners. While the ANDS provided an overall country strategy towards development, including nutrition, food security and WASH actions, government-wide efforts to recognise nutrition as foundational to development, establish nutrition targets for which all related sectors are responsible, and identify feasible actions to achieve nutrition targets were only initiated in 2012.

While nutrition requires multisectoral actions, in the Afghanistan context, it is more realistic for institutions to initially build capacity by sector because integrated or multisectoral service delivery requires stronger institutional capacity [2]. Institutional capacities required to effectively mount a multisectoral response to malnutrition include sufficient capacity to undertake policy-making, programme design, implementation, training, monitoring, and changing perceptions and understanding malnutrition [28]. Two key documents developed in 2012 established nutrition as a government priority, the Nutrition Action Framework and the Afghanistan Food Security and Nutrition Agenda (AFSANA, changed to AFSeN in April 2017). AFSeN provides the government's policy statement affirming its commitment and determination to have a coordinated response to the underlying determinants of hunger and malnutrition. While it is a comprehensive policy document, there has been relatively low operationalisation to date by line ministries. By 2015, three of the five key nutrition, food security, and WASHrelated policy and strategy documents were developed with government-wide support between the MoPH, Ministry of Rural Rehabilitation and Development, MAIL, and the Ministry of Education [29-31]. In 2018, an advocacy and information strategy and an implementation plan were developed as a key step toward AFSeN strategy functionality [32]. The plan was developed by a multi-agency working group comprising representatives from the MoPH, MAIL, Ministry of Education, Central Statistics Office, Ministry of Information and Cultural Affairs, Ministry of Women's Affairs, Ministry of Energy and Water, Afghan National Standards Authority, Afghanistan Human Rights Organization and international partners.

All policy and strategy documents referenced the most recent descriptive statistics, relying heavily on the 2013 National Nutrition Survey (NNS) and the 2017 National Risk and Vulnerability Assessment (NRVA)/Afghanistan Living Conditions Survey (ALCS), but less commonly referenced peer-reviewed statistical analyses of predictors of nutrition outcomes. There was a focus on using national level statistics, even when disaggregated provincial or regional statistics were available. While variation in nutrition status across provinces was recognised and the need for context-specific strategies was often acknowledged, provincial or regional estimates were not cited or used for strategy development or for evaluating changes in nutrition status over time. Recent policies 
and strategies emphasised and defined multisectoral inclusion by the line ministry, especially when discussing intervention strategies across sectors and coordinating among ministries and implementing partners. However, the integration of intervention strategies was less detailed in these documents. Programme strategy responses between 2004 and 2009 focused on the treatment and prevention of malnutrition through health service delivery, control of micronutrient deficiencies through supplementation, and IYCF practices. While social and behaviour change programming, treatment guidelines and community-based nutrition services (e.g. management of moderate acute malnutrition (MAM), community-based management of acute malnutrition (CMAM) pilots and integrated community case management) were introduced prior to 2013, the more recent strategies focused on continued quality improvement, integrated strategies (integrated management of acute malnutrition (IMAM) in 2014), and taking these services to greater scale [33]. Additionally, food quality, safety and standards, and expanding nutrition-sensitive food products in agriculture programmes were frequently mentioned, though linkages to health programmes were limited. Further, it was unclear whether integrating nutrition with other health programmes occurred at the implementation level because, while strategies highlighted multipronged or multisectoral approaches, they were not explicit regarding the level of integration. Table 2 provides a summary of all relevant policies and strategies.

\section{Assessing the nutrition situation in children: data sources Overall nutrition status}

Stunting of children $0-59$ months was $40.9 \%$ in 2013, with severe stunting at $20.9 \%$ and moderate stunting at $20.0 \%$ [34]. Stunting prevalence varied from $24.3 \%$ to $70.8 \%$ by province. While still high, these numbers represent a decrease in national stunting rates from $60.5 \%$ in 2004 [35]. Micronutrient deficiencies were also pervasive among women of reproductive age and children 659 months of age. There is evidence of low dietary diversity among all Afghans, particularly among children under 2 years of age. In 2015, just under a quarter of children under 2 years of age had been given foods from the minimum number of food groups and half were fed at least the minimum number of times appropriate for their age [36]. Overall, correct IYCF practices, particularly exclusive breastfeeding to 6 months and frequency of complementary feeds, remained low and there was a disparity between rural and urban areas in the percentage of children aged 6-23 months fed according to IYCF guidelines (13\% vs. $22 \%$, respectively) [36]. Only $58.9 \%$ of children under 5 years lived in households using iodised salt, which is far below the $90 \%$ recommendation for household coverage to eliminate iodine deficiency [36, 37].

Expansion of access to improved drinking water and sanitation facilities has been slow. In 2015, over one-third of the population did not have access to an improved source of drinking water and threequarters did not have access to an improved sanitation facility [34, 36, 38]. In 2013, the percentage of women who reported handwashing (89.7\%) exceeded the observed available handwashing places in the home (45.1\%) [34].

\section{Overview of key national data sources}

Since 2002, nutrition data within Afghanistan has increased (Table 3). Overall, while more surveys have collected nutrition-related information from households since 2012, there has also been a significant increase in the analysis of population-based survey data to better understand the factors associated with poor nutrition practices and outcomes. Prior to 2004, evidence on child nutrition status was confined to small area surveys specific to informing small-scale health and nutrition programmes or hospital surveys based on disease-specific cases with malnutrition as a secondary outcome or additional risk factor for mortality. The first NNS [39] was conducted in 2004 and provided the only comprehensive national nutrition assessment; however, provincial level estimates were not available due to the sampling methodology. In 2013, another NNS was conducted to provide both national and provincial level representative estimates for key nutrition indicators, except for micronutrient-related deficiencies due to the limited sample collection. The 2013 NNS [34] also included a qualitative knowledge, attitudes and practices component on IYCF and on prevention of micronutrient deficiencies. We use the two NNS as time bounds to understand the generation of nutrition-related data before, during and after they were conducted, given the impact of these data on nutrition policy and programming (Table 3).

The Afghanistan Living Conditions Survey [40] (ALCS, previously known as the NRVA) was most recently conducted in 2016-2017 and provides multisectoral estimates on poverty, food security, education, health, labour market, agriculture and other critical indicators. The NRVA/ALCS has been implemented routinely, generally at 2-3-year intervals, since 2003. The Multiple Indicator Cluster Survey (MICS) was conducted four times, three of which were before 2004 . The MICS is supported by UNICEF and focuses on child rights and development indicators. Two health sector-specific surveys are the Afghanistan Health Survey (AHS) [41] and the Afghanistan Demographic and 


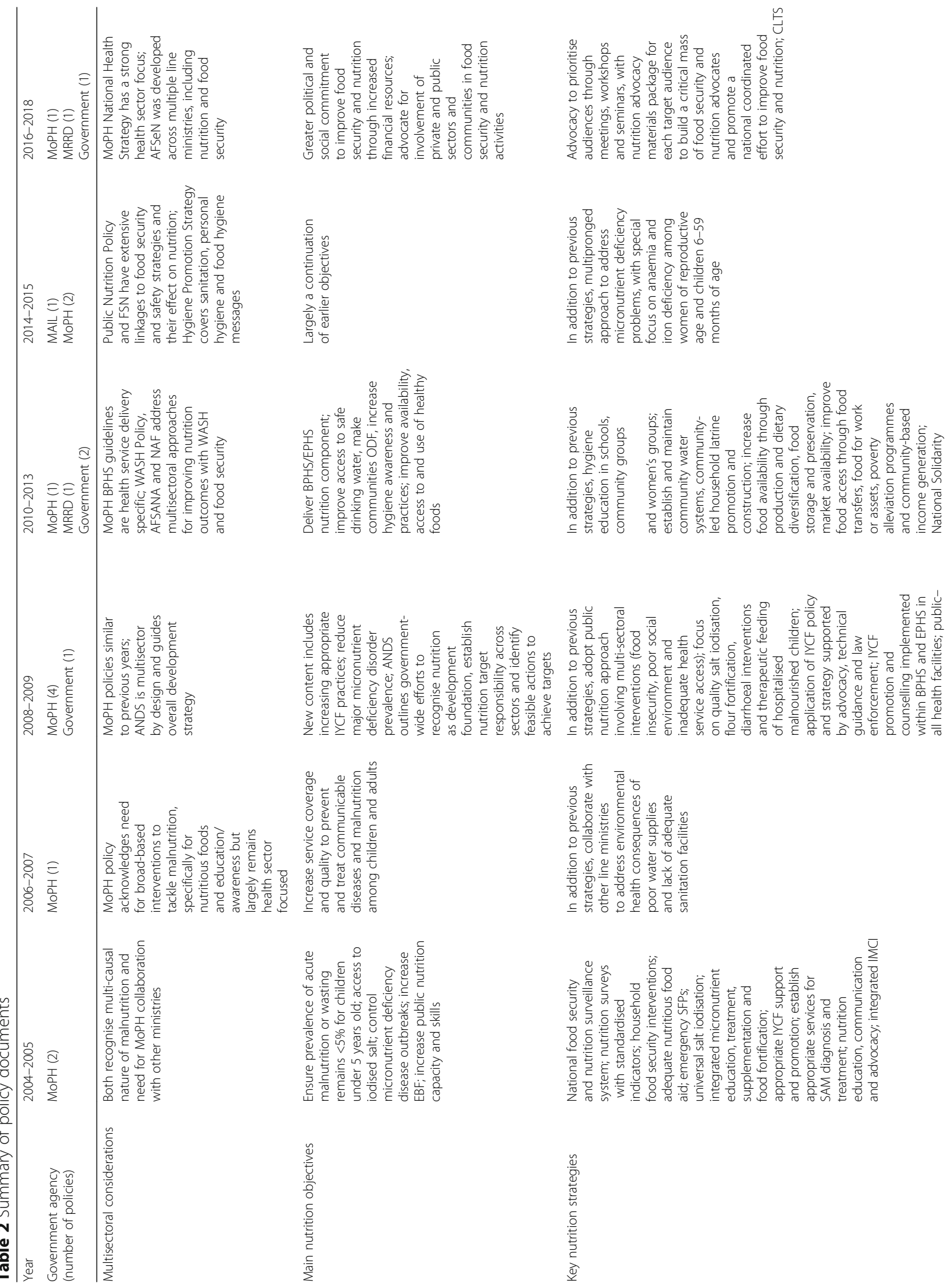




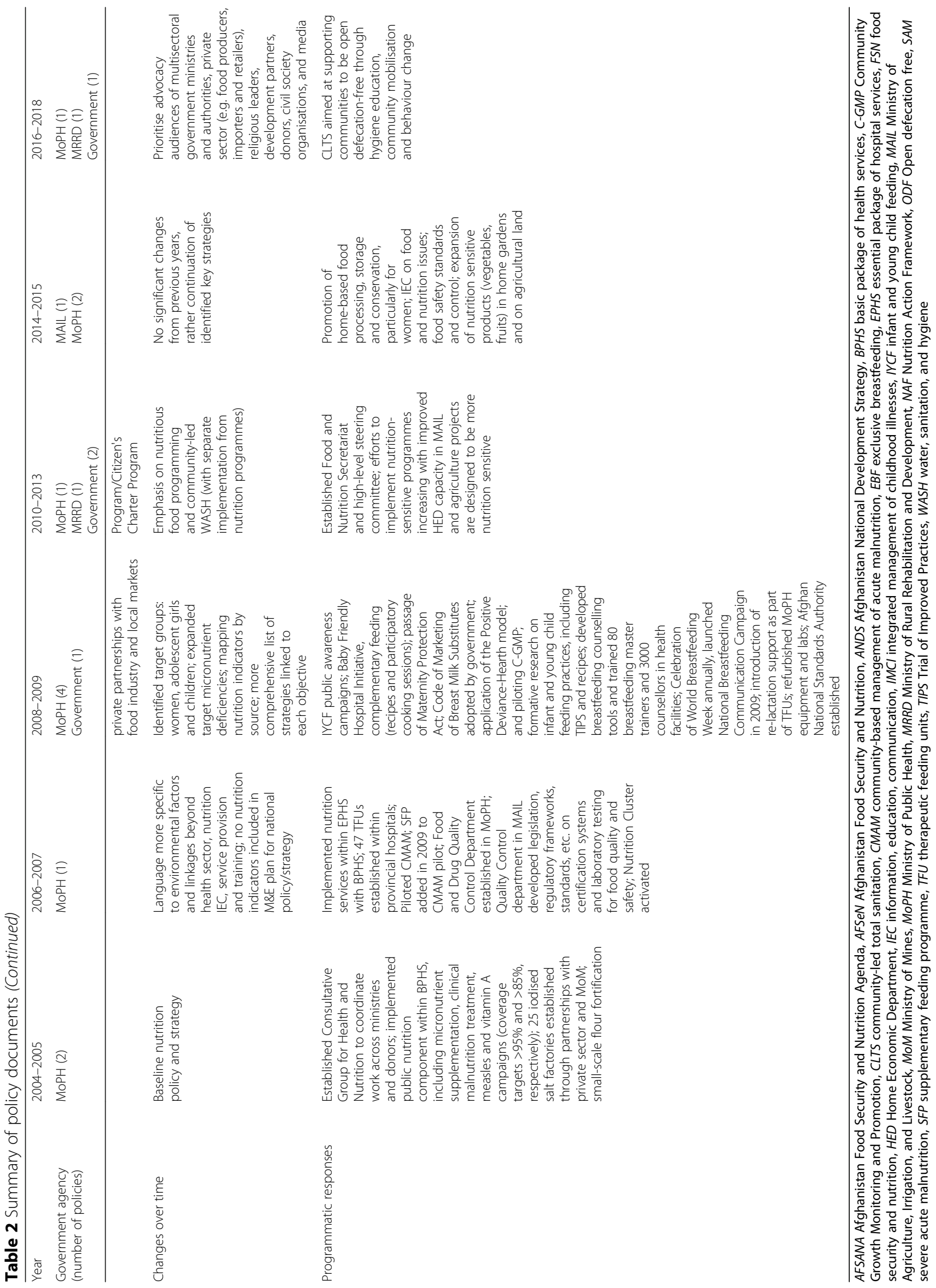


Table 3 Summary of data sources with nutrition-related measures

\begin{tabular}{|c|c|c|c|c|}
\hline & & 2004 NNS and earlier & $2005-2012$ & 2013 NNS and later \\
\hline Number of data sources & & 15 & 12 & 18 \\
\hline \multirow[t]{3}{*}{ Type of data collection } & Primary data collection reports & 15 & 10 & 13 \\
\hline & Secondary data analysis & 0 & 1 & 4 \\
\hline & Use of both primary and secondary data & 0 & 1 & 1 \\
\hline \multirow[t]{4}{*}{ Geographic representation } & National & 1 & 2 & 4 \\
\hline & National and/or Regional & 2 (2 regional only) & 1 & 1 \\
\hline & National and/or Provincial & 0 & 3 & $\begin{array}{l}5 \text { ( } 4 \text { single-province/ } \\
\text { district/urban areas only) }\end{array}$ \\
\hline & $\begin{array}{l}\text { Specific area to programme/facility and/ } \\
\text { or not representative of any region }\end{array}$ & 10 & 6 & 4 \\
\hline \multirow[t]{3}{*}{ Implementers } & NGO & 11 & 3 & 6 \\
\hline & Government/CSO & 1 & 4 & 4 \\
\hline & $\begin{array}{l}\text { Academic institution/Research organisation } \\
\text { (National or Int'l) }\end{array}$ & 3 & 5 & 8 \\
\hline \multirow[t]{30}{*}{ Child nutrition-related measures } & IYCF & & & \\
\hline & Ever breastfed & 3 & 1 & 2 \\
\hline & Early initiation of breastfeeding & 3 & 2 & 5 \\
\hline & Use of pre-lacteal feed & 1 & 0 & 2 \\
\hline & Discarded colostrum & 2 & 0 & 1 \\
\hline & Exclusive breastfeeding & $3 \mathrm{~m}(1) ; 4 \mathrm{~m}(2) ; 6 \mathrm{~m}(1)$ & $5 \mathrm{~m}(2) ; 6 \mathrm{~m}(2)$ & 5 m (2); 6 m (4) \\
\hline & Complementary foods & $6-9$ m (4) & $6-9$ m (4) & 6-8 m (4); 6-9 m (1) \\
\hline & Minimum acceptable diet (4+ groups) & 0 & 0 & 2 \\
\hline & Minimum meal frequency & 0 & 1 & 2 \\
\hline & Continued breastfeeding at 1 year & 3 & 1 & 3 \\
\hline & Micronutrients & & & \\
\hline & $\begin{array}{l}\text { Vitamin A supplementation/ } \\
\text { deficiency/night blindness }\end{array}$ & 4 & 6 & 4 \\
\hline & $\begin{array}{l}\text { lodised salts/visible goitre/iodine } \\
\text { deficiency disorders }\end{array}$ & 4 & 3 & 2 \\
\hline & Anaemia/iron deficiency & 1 & 2 & 1 \\
\hline & Zinc deficiency & 0 & 0 & 1 \\
\hline & Vitamin D deficiency & 0 & 1 & 1 \\
\hline & Vitamin C deficiency & 1 & 0 & 0 \\
\hline & Immunisations & & & \\
\hline & Measles & 4 & 5 & 3 \\
\hline & Fully immunized & 2 & 2 & 2 \\
\hline & PENTA 3 & 0 & 1 & 1 \\
\hline & WASH & & & \\
\hline & Safe drinking water & 4 & 4 & 6 \\
\hline & Household water insecurity & 1 & 0 & 0 \\
\hline & Improved sanitation & 5 & 4 & 7 \\
\hline & Handwashing with soap/ash, at key times & 1 & 1 & 5 \\
\hline & Food security & & & \\
\hline & Sufficient food last week & 1 & 0 & 0 \\
\hline & Household perception of food security & 0 & 2 & 0 \\
\hline & Dietary diversity & 0 & 1 & 3 \\
\hline
\end{tabular}


Table 3 Summary of data sources with nutrition-related measures (Continued)

\begin{tabular}{|c|c|c|c|c|}
\hline & & 2004 NNS and earlier & $2005-2012$ & 2013 NNS and later \\
\hline & Calorie deficiency & 0 & 1 & 1 \\
\hline & Protein deficiency & 0 & 1 & 1 \\
\hline & Hunger scale & 0 & 0 & 1 \\
\hline & Food insecure population & 0 & 0 & 2 \\
\hline & Households receiving food aid & 1 & 0 & 0 \\
\hline & Households owning garden plot & 0 & 0 & 3 \\
\hline & $\begin{array}{l}\text { Acceptable food consumption/ } \\
\text { diet, coping mechanisms }\end{array}$ & 1 & 0 & 2 \\
\hline & Childhood illness & & & \\
\hline & Diarrhoea in 2 weeks preceding survey & 5 & 4 & 3 \\
\hline & ARI in 2 weeks preceding survey & 4 & 3 & 2 \\
\hline & $\begin{array}{l}\text { Other illnesses related to malnutrition } \\
\text { or outcomes affected by malnutrition }\end{array}$ & $\begin{array}{l}\text { Pyogenic meningitis (1) } \\
\text { Measles (3) }\end{array}$ & 0 & 0 \\
\hline & Nutrition outcomes & & & \\
\hline & MUAC GAM/MAM/SAM & 4 & 1 & 5 \\
\hline & $\begin{array}{l}\text { Wasting/MAM/SAM (weight for } \\
\text { height <-2 SD) }\end{array}$ & 4 & 3 & 3 \\
\hline & Underweight (weight for age <-2 SD) & 3 & 2 & 3 \\
\hline & Stunting (height for age $<-2$ SD) & 4 & 3 & 3 \\
\hline & Overweight (weight for height >+2 SD) & 0 & 0 & 1 \\
\hline & Low birth weight & 0 & 0 & 1 \\
\hline \multirow{2}{*}{$\begin{array}{l}\text { Sources without nutrition } \\
\text { indicators }\end{array}$} & Qualitative study & 0 & 0 & 2 \\
\hline & $\begin{array}{l}\text { Statistical analyses on variables } \\
\text { associated with nutrition outcomes }\end{array}$ & 0 & 0 & 4 \\
\hline
\end{tabular}

ARI acute respiratory infection, CSO Central Statistics Organization, GAM global acute malnutrition, IYCF infant and young child feeding, $m$ months, MAM moderate acute malnutrition, MUAC mid-upper arm circumference, NGO non-governmental organisation, NNS National Nutrition Survey, PENTA pentavalent vaccine (diphtheria, pertussis, tetanus, hepatitis B, Haemophilus influenza), SAM severe acute malnutrition, SD standard deviation, WASH water, sanitation, and hygiene

Health Survey (ADHS) [42]. The AHS, implemented since 2006, is an annual survey that provides national and provincial level estimates on priority health sector indicators and is used to evaluate the health service delivery projects, most recently the System Enhancement for Health Action in Transition. The ADHS was first implemented in 2015 to provide national and provincial level estimates on a range of demographic and health indicators collected in other low- and middle-income countries. Programme-related rapid nutrition assessments have been conducted more recently (Standardized Monitoring and Assessment of Relief and Transitions (SMART) surveys [43-45] and Semi-Quantitative Evaluation of Access and Coverage (SQUEAC) [46, 47]). These assessments aim to provide rapid coverage estimates and programme area information on population nutrition status at the subnational level. All aforementioned surveys are cross-sectional in design. Finally, the national nutrition surveillance bulletin provides quarterly surveillance information across 175 facility and 953 community sentinel sites [48].
Survey implementation varied between government agencies (mainly the Central Statistics Office), academic or research institutions, and NGOs. Child nutritionrelated measures are organised into eight categories, namely IYCF, micronutrients, immunisations, mid-upper arm circumference (MUAC), WASH, food security, childhood illness and nutrition outcomes. Duplication of data collection was minimal during each time period as no data sources collected identical nutrition indicators. We incorporated commonly reported nutrition-related indicators into the IYCF practices, immunisation and WASH categories.

\section{Nutrition outcomes}

Anthropometric measures have been used to estimate the prevalence of malnutrition from before 2002. These measures were obtained during household surveys and nutrition status was assessed using a MUAC as well as weight-for-height (wasting), weight-for-age (underweight) and height-for-age (stunting), though not all indicators have been used consistently across studies. 
The prevalence of the overweight population was not assessed until the 2013 NNS. MUAC was more widely used across national-level household surveys and among programme area assessments of nutrition status. Most data sources did not measure nutrition outcomes; only the MICS and NNS reported on wasting, stunting and underweight. Small area surveys reported global acute malnutrition and severe acute malnutrition estimates. Methodological difficulties capturing anthropometric data in this context compromised obtaining children's precise age and height, including a representative sample of vulnerable populations, and limited reference data for infants less than 6 months old [49].

\section{Micronutrient deficiencies}

Vitamin A supplementation and presence of household iodised salt have been consistently monitored over time. Reported micronutrient deficiency data sources prior to 2004 were based on small-scale surveys and assessed clinical signs of diseases such as scurvy [50]. In the 2004 NNS, $81 \%$ of children aged 6-59 months received vitamin A supplementation and $28.3 \%$ of households were found to have any iodine in their salt [39]. Anaemia and iron deficiency anaemia were also measured; $37.9 \%$ of children aged $6-$ 59 months were anaemic and $33.4 \%$ had iron deficiency. In the 2013 NNS, $44.6 \%$ of children under 5 years received vitamin A supplementation, $44.9 \%$ were anaemic and 13.7\% had iron deficiency. The 2013 NNS also collected data for vitamin D (81\%) and zinc deficiency $(15.1 \%)$ in the same age group.

\section{Infant and young child feeding practices}

Evidence on optimal breastfeeding practices in Afghanistan shows decreasing prevalence of key IYCF indicators, including early initiation of breastfeeding within 1 hour of birth, exclusive breastfeeding (EBF) for the first 6 months, and continued breastfeeding up to 2 years of age with appropriate complementary foods [23]. These practices may have always been poor as documented by a 1983 study of infant feeding practices in Kabul [51]. This study captured the traditional practice of pre-lacteal feeds, such as butter or tea, early breastfeeding cessation due to perceived insufficient milk supply, and poor introduction of complementary foods [51]. Most data sources have not collected all IYCF indicators; rather, only one or two breastfeeding-related indicators are reported. Further, the age ranges used for EBF and continued breastfeeding measures have been inconsistent across sources [34, 36, 41, 52].

The transition from EBF to solid foods is a critical period for children as they are most vulnerable to becoming undernourished during this time. Minimum acceptable diet and minimum meal frequency are relatively new indicators that serve as proxies for adequate micronutrient density of foods and energy requirements, respectively. These have only been collected by recent large national health surveys. The 2015 ADHS found that only $16 \%$ of children aged 6-23 months received the minimum acceptable diet [36]. Qualitative studies describing IYCF practices and beliefs around infant feeding have only been conducted since the 2013 NNS. These studies highlighted traditional practices and regional preferences for pre-lacteal feeds and the rejection of colostrum often instructed by mothers-in-law [34], the perceived need to bathe an infant before initiating breastfeeding [53] and perceptions around low milk supply [34, 54].

\section{Healthcare and environment}

Indicators for immunisation (immunised for measles or fully immunised by 24 months), WASH (safe drinking water and improved sanitation) and childhood illnesses (diarrhoea and acute respiratory infection) were more widely collected across health and non-health data sources. The ADHS found that $46 \%$ of children were fully vaccinated, $65 \%$ of households had access to an improved drinking water source, $25 \%$ of households had access to improved sanitation facilities, and 29\% of children had diarrhoea 2 weeks before the survey [36]. Since 2013, more data have been collected on caregiver (generally mothers) handwashing practices at critical times (47.5\% of mothers) such as before feeding a child and after disposing of a child's faeces [55]. One study found that, in urban areas, only 33\% of respondents reported handwashing before eating and only $21 \%$ of female respondents reported handwashing before food preparation [56].

\section{Food security and dietary diversity}

Before 2013, there was less information available regarding underlying causes of malnutrition related to food security, livelihoods and dietary diversity. While efforts were made to integrate nutrition information into wider vulnerability assessments, such as the NRVA, food security indicators have not been consistently defined or collected. Food security and dietary diversity measures ranged across different sectors, with the food security situation predominantly described qualitatively. Overall poor access to diverse foods and low dietary diversity has been documented since before 2004; vulnerability to food insecurity was documented as a mixture of households' ability to cope with economic risks, socio-political and geographic risks, natural and manufactured hazards, and risks due to food aid delivery [57]. The 2005 NRVA estimated that $24 \%$ of households had low dietary diversity and $44 \%$ perceived themselves to be food insecure. The 2014 ALCS estimated that 33\% of the population 
was food insecure. These values are not comparable due to differences in indicator definitions. Levitt et al. [58] found that food availability is generally not the main concern in Afghanistan; rather, dietary diversity is limited due to poor economic ability to access a variety of available foods.

\section{Secondary data analyses}

Secondary data analyses have increased since the 2013 NNS as more national and provincial level representative sample surveys were conducted. These studies have assessed trends in IYCF indicators and associated factors [23], geographic disparities in nutrition status and factors associated with stunting and underweight [59], factors associated with poor complementary feeding practices [60], and the relationship between irrigation and dietary diversity [61]. Benedict et al. [23] found that all positive IYCF indicators have decreased significantly; for example, EBF significantly decreased by 10 percentage points from 2010 to 2016 . Large district-level disparities exist in child nutrition outcomes such as stunting prevalence ranging from $4 \%$ to $84 \%$ [59]. A greater number of antenatal care visits and increasing child age were positively associated with meeting all complementary feeding practices [60]. Irrigation facilities were found to be important and positively correlated with diversity of food intake from households' own production and garden plots were positively associated with greater diversity of food purchased at markets [61].

\section{Presenting summary evidence for decision-making}

The presentation of results and recommendations based on the generated data are important to guide policymakers and other stakeholders to make evidence-based decisions. Survey reports generally included an executive summary with a key indicators table. Policy recommendations based on results differed among reports and most reports did not include their key recommendations in the executive summary. More recent studies have included trends in key indicators using several data points such as the AHS 2015 and the ALCS 2016-2017. However, most studies cautioned against comparisons with previous estimates due to differences in methodology and indicator definitions as well as limitations of data collection and representativeness. A commonly cited data limitation was the lack of representation of insecure clusters, as these often get dropped and/or more secure clusters are over sampled.

\section{Nutrition programme responses and multisectoral approaches}

We found 20 eligible intervention studies matching inclusion criteria (Table 4). The studies ranged in publication dates from 1986 to 2017, 14 of which were published in or after 2013. However, all but two interventions were implemented prior to 2013. Most studies explicitly targeted children under 5 or 2 years of age and studies of household interventions specifically targeted women and young children. Interventions ranged from nutrition awareness and health promotion activities [62, 63, 75, 79], micronutrient deficiency treatment or prevention [50,65-67, 80], malnutrition treatment or prevention [68, 70-72, 77, 78], and delivery or modelling of a package of community and facility-based interventions, including multisectoral approaches $[64,69,74,76,81]$.

\section{Nutrition awareness and health promotion}

Nutrition awareness is often promoted through campaigns on different topics such as breastfeeding, hygiene, growth monitoring and complementary feeding at group education sessions [63, 71, 81], training for women and households participating in kitchen gardens and select agriculture activities [81], and other targeted projects. These tend to be topic and agenda specific by project. Almost all interventions included an education component, with four studies also focused on raising awareness and promoting health and nutrition messages. These were done through group education for mothers on child growth charts [62], interactive electronic books with public health messages [63], a nutrition and hygiene awareness pilot as part of an unconditional cash transfer project [79], and brochures on IYCF best practices for newborns [75]. These nutrition awareness programmes all included mothers of young children as the target audience, despite pilot study findings of additional benefit in targeting men or mothers-in-law (or other influential family members) with tailored messages [79]. All studies reported some improvement in knowledge levels of participants, but these were either qualitative feedback from participants or sample sizes were generally small and statistical tests were not often used to compare preand post-intervention changes. One study noted that understanding the importance of breastfeeding was high but the timing for complementary food introduction and handwashing before feeding children were frequently misunderstood [79]. Only one study assessed selfreported IYCF practices and found no differences between baseline and endline in early initiation of breastfeeding [75].

\section{Prevention and treatment of micronutrient deficiencies}

There were four types of programmes designed to reduce micronutrient deficiencies in Afghanistan - food diversification, micronutrient supplementation, food fortification and micronutrient deficiency disorder treatment [2]. Four studies evaluated micronutrient deficiency disorder treatment, specifically vitamin $C$ to treat scurvy [50], and 


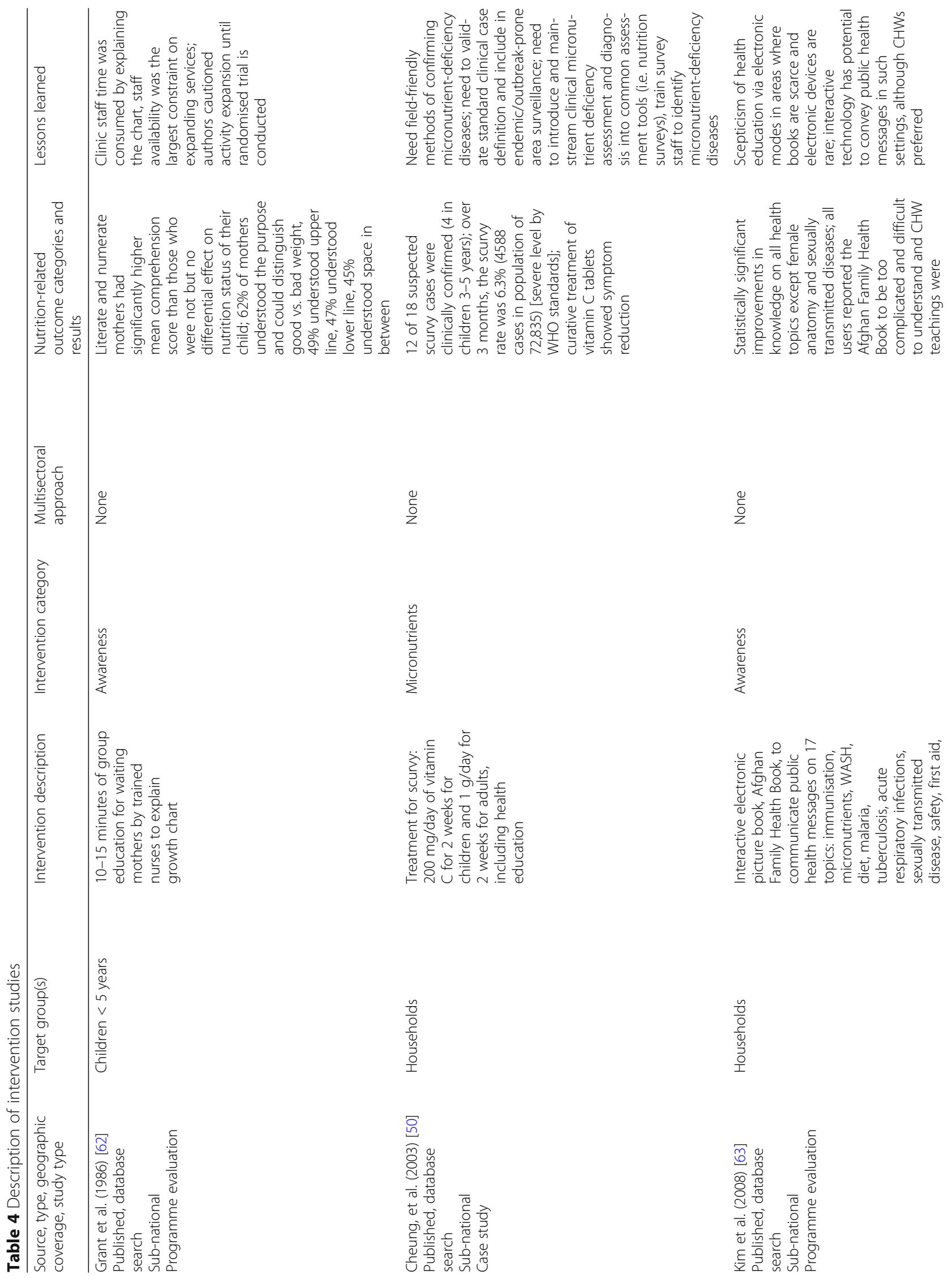




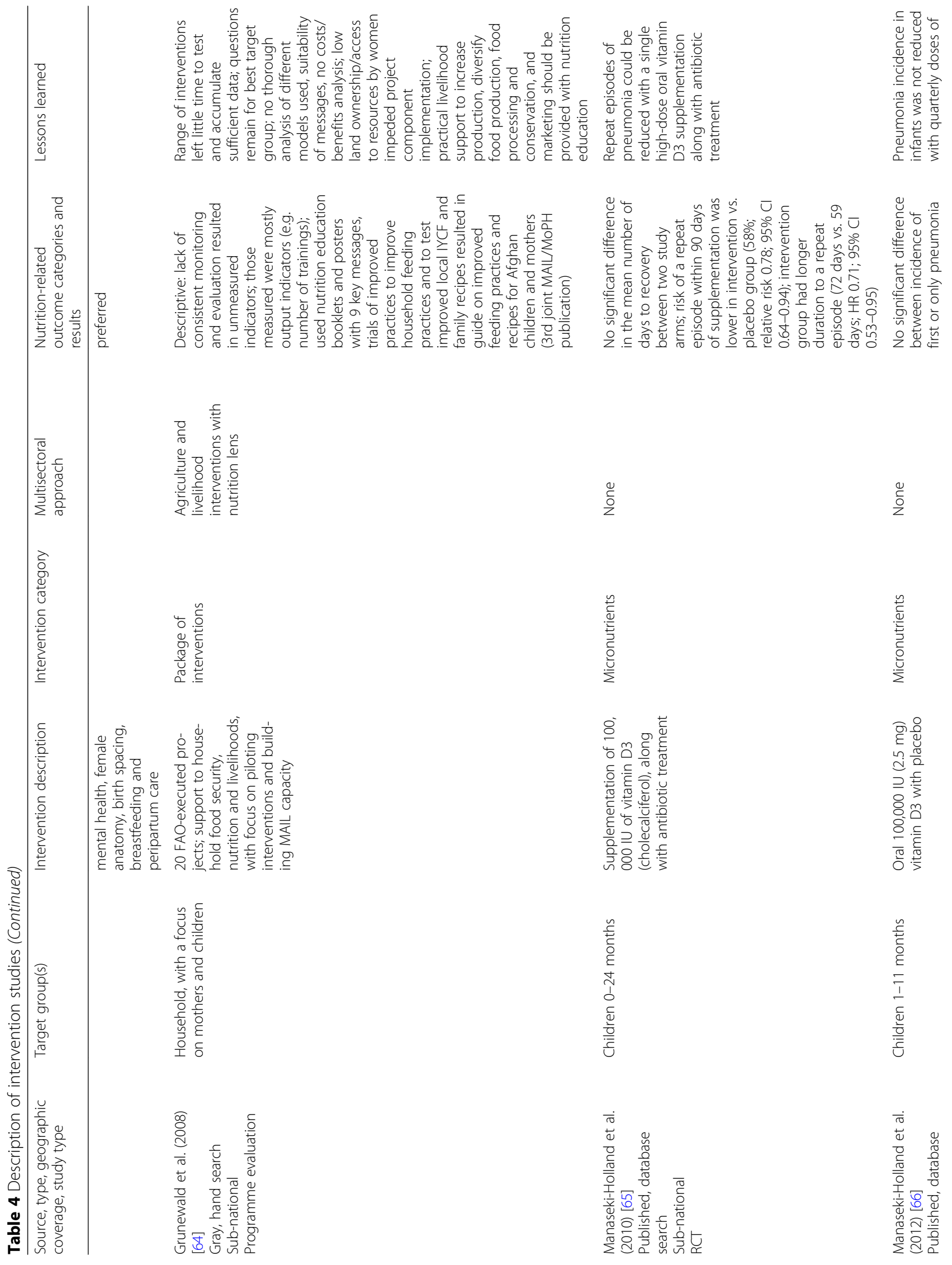




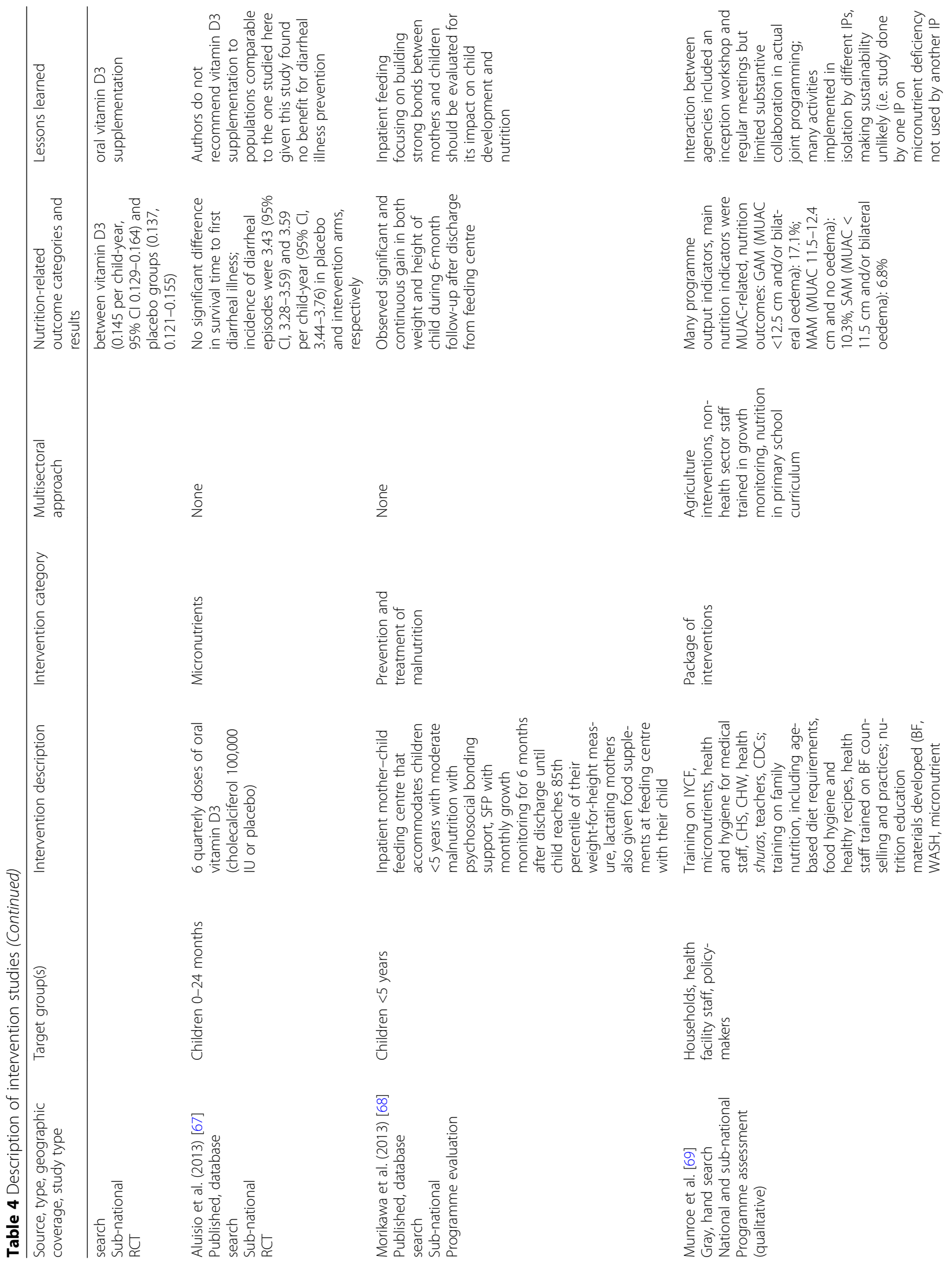




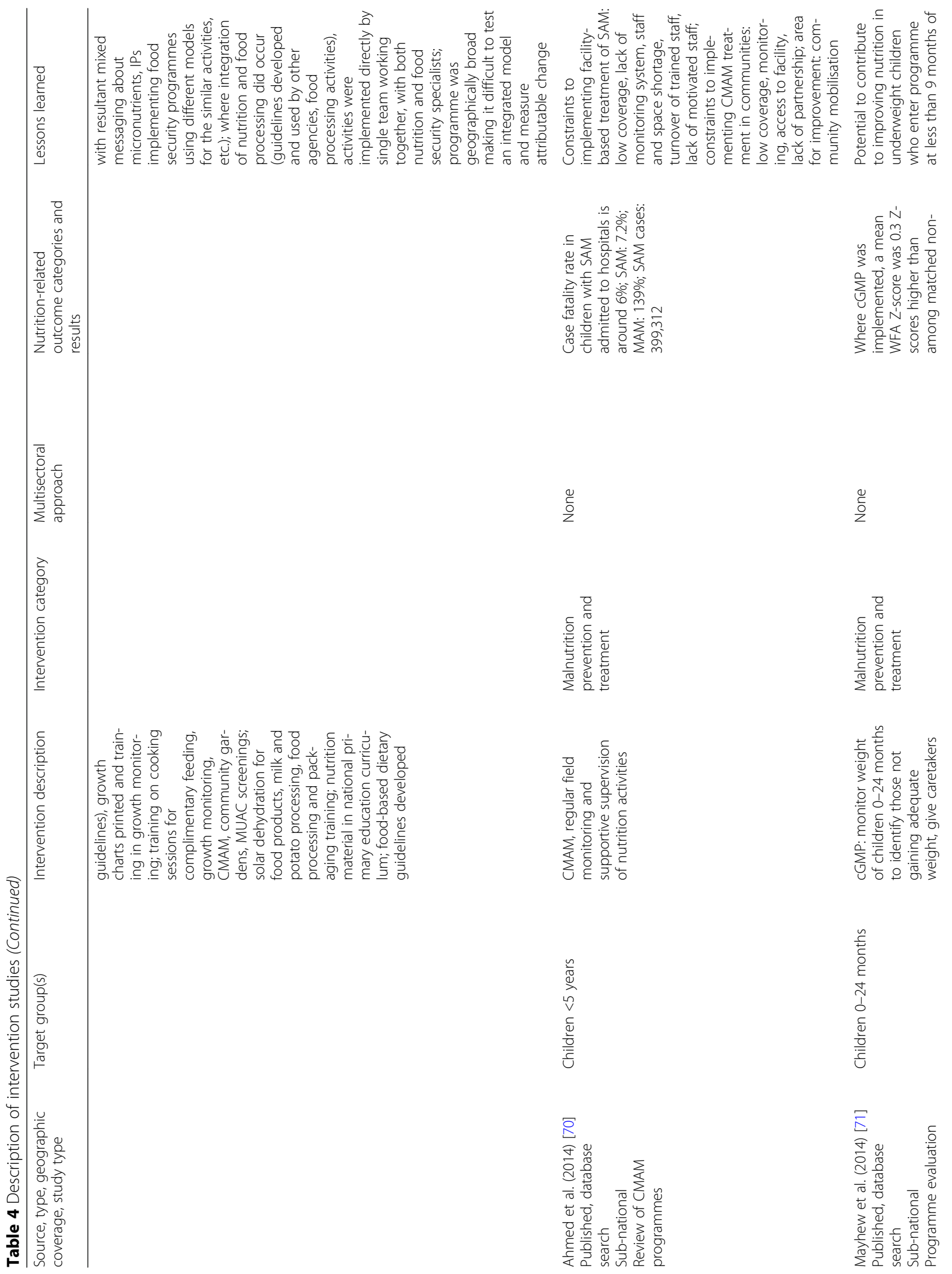




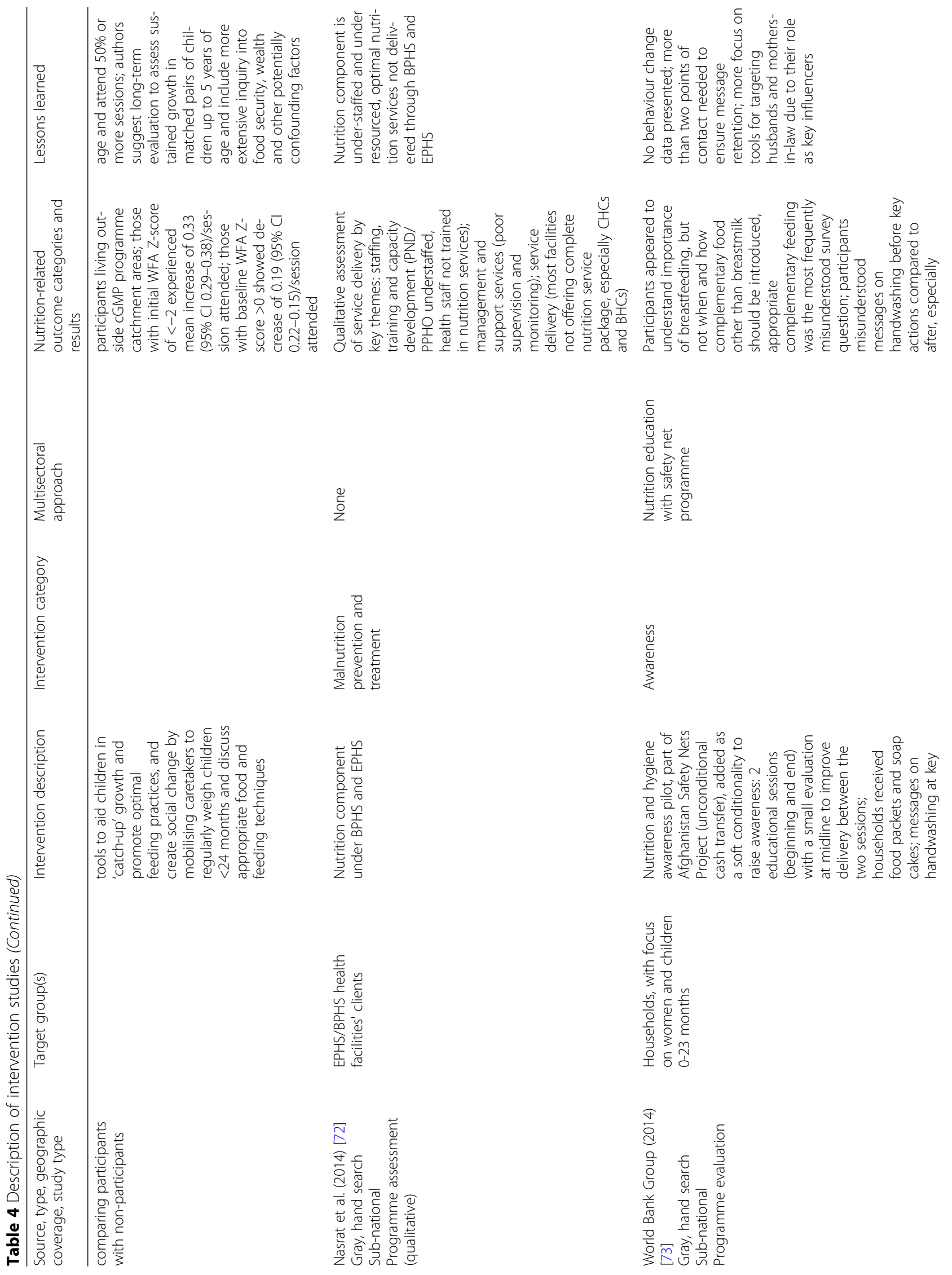




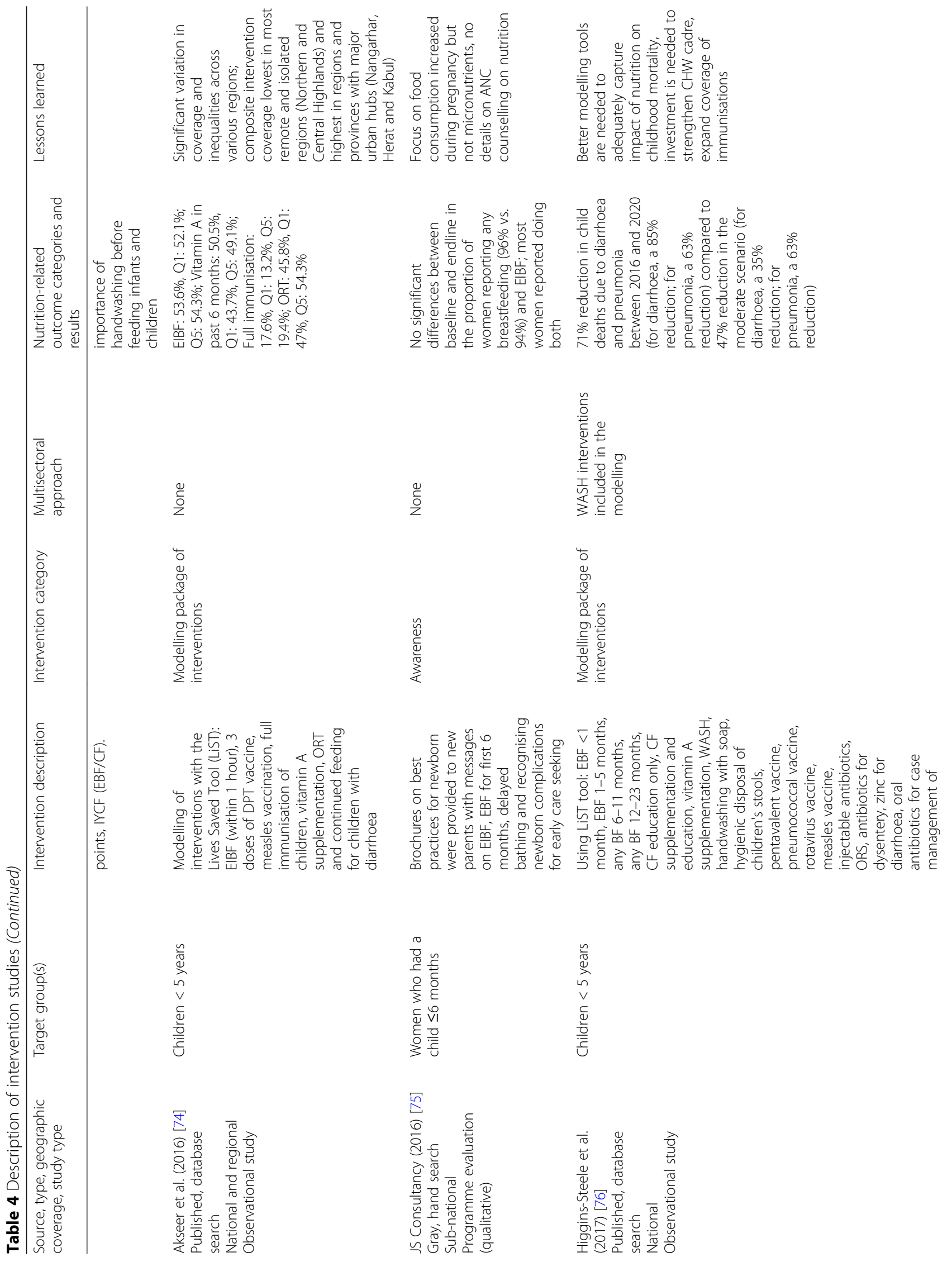




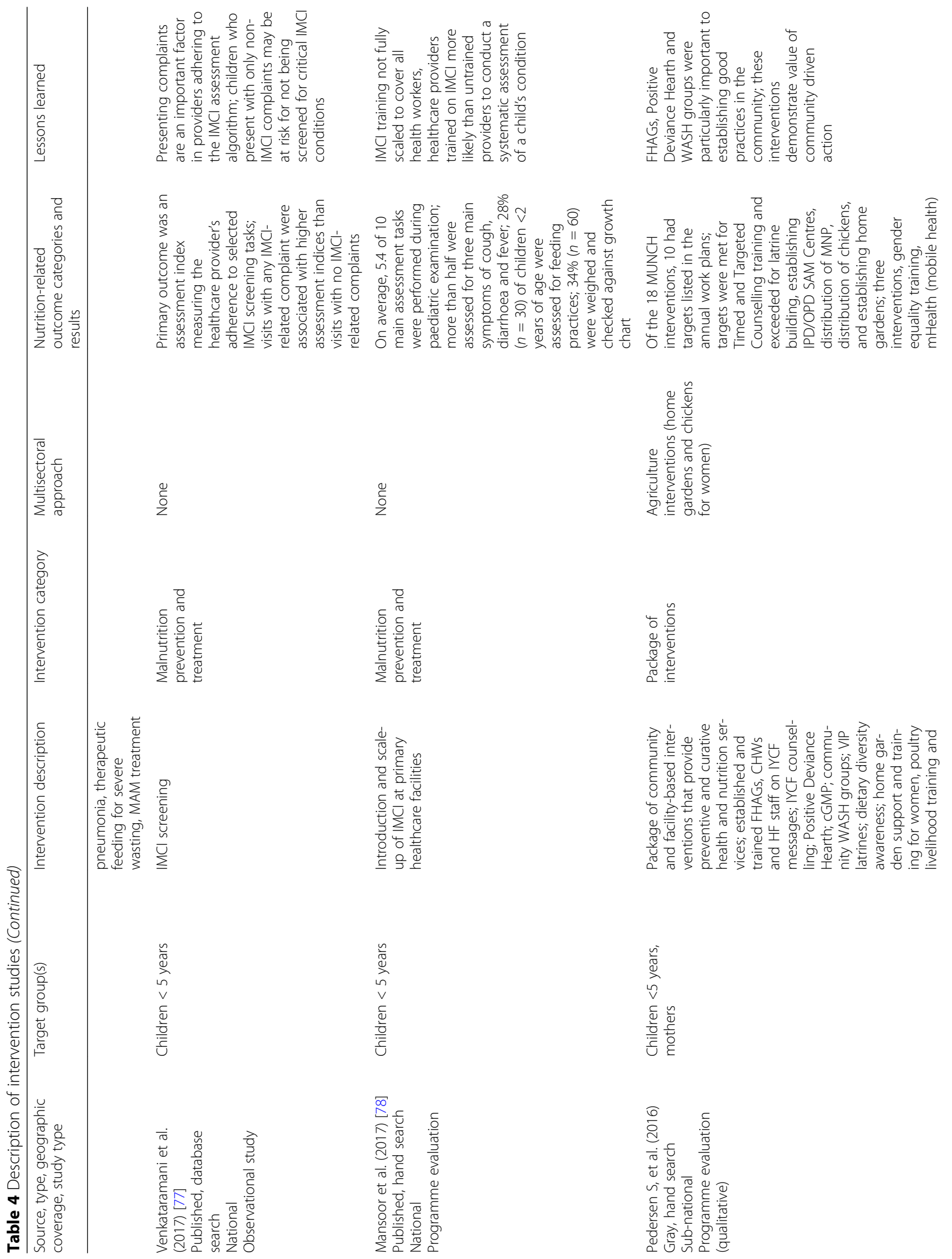




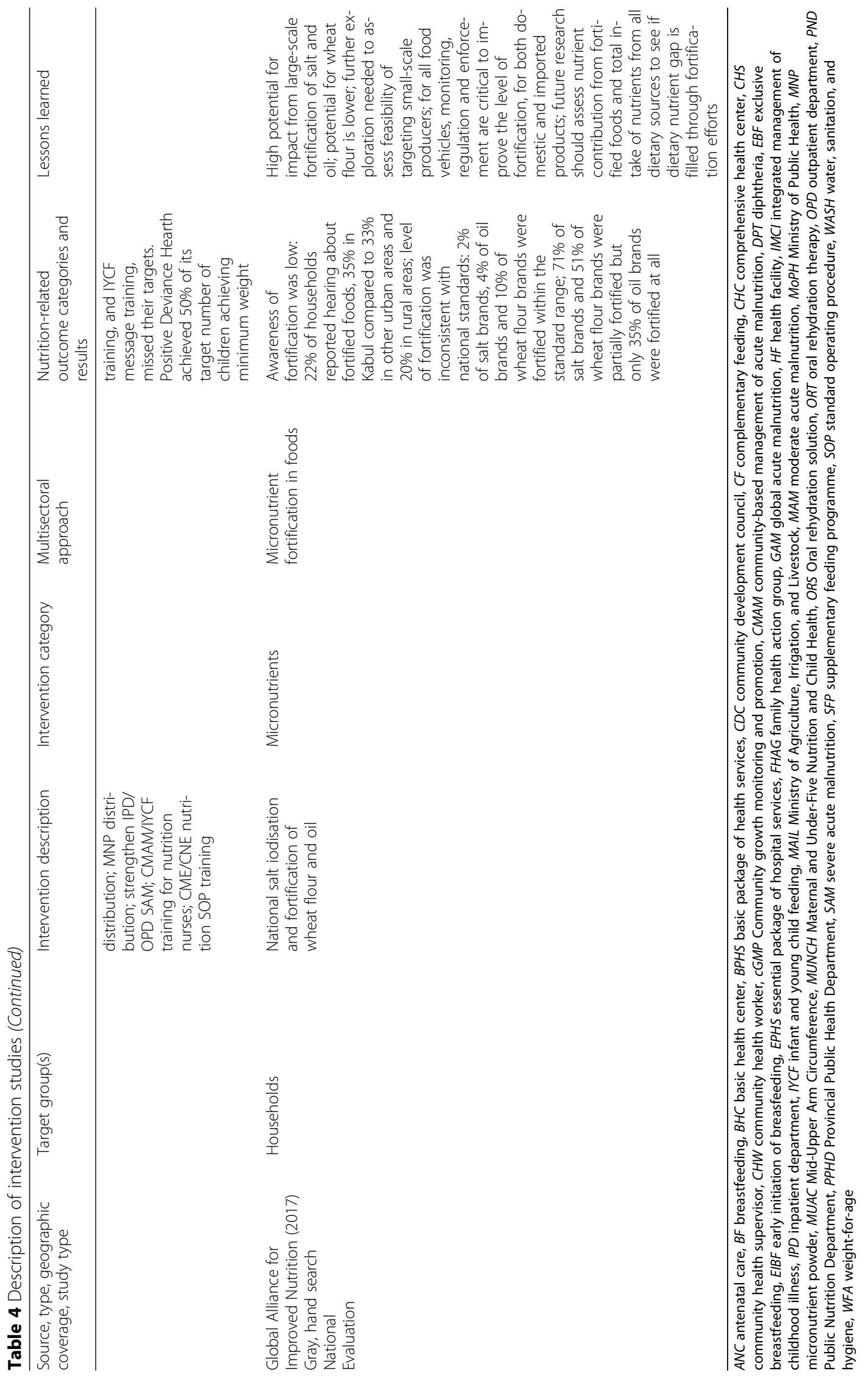


micronutrient supplementation, specifically vitamin D3 to reduce the duration and occurrence of pneumonia [65, 66], and to decrease the incidence of diarrhoea in children under 5 years [67]. Vitamin D3 was found to be ineffective for reducing the incidence of either diarrhoea or pneumonia in the studied population but was effective for reducing recurrent episodes of pneumonia when taken with antibiotics.

One study assessed national food fortification coverage and found that the level of fortification was below national standards: only $2 \%$ of salt brands, $4 \%$ of oil brands and $10 \%$ of wheat flour brands were fortified within the standard range [80]. Household consumption of fortified food was also low; only $22.1 \%$ of households consumed fortified salt, $30.1 \%$ consumed fortified oil and $18.6 \%$ consumed fortified wheat flour [80]. The low availability and consumption of fortified wheat flour is likely due to production and milling through small-scale systems that do not provide fortification, used by more than $80 \%$ of rural households [82].

At the facility level, micronutrient supplementation is provided by midwives, vaccinators and doctors. Midwives provide iron and folic acid supplements for pregnant and lactating women, vaccinators provide vitamin A supplements for children 6-59 months of age, and doctors provide iron for low birth-weight babies and vitamin $\mathrm{C}$ for populations at high risk. An assessment of the Basic Package of Health services (BPHS) and the Essential Package of Hospital Services (EPHS) nutrition components found that, while health workers are tasked with promoting fortified food consumption, such as iodised salt, and community health workers ( $\mathrm{CHWs}$ ) are tasked with including discussions on fortified foods at community health shura (community leadership committee) meetings, many households likely do not have access to fortified flour and oil [72]. Additionally, CHWs are supposed to provide micronutrient powders for home-based fortification for all children under 2 years of age; however, micronutrient powders are not on the essential drug list for health posts in the BPHS. Additionally, the BPHS essential drug list does not include multimicronutrient vitamins at the health post (community) level. Thus, these supplies are often not available at community level, unless supplied via an external project.

\section{Prevention and treatment of malnutrition and childhood illnesses}

The Integrated Management of Childhood Illness (IMCI) programme was adopted globally as a strategy to reduce morbidity and mortality in children under 5 years of age [83]. IMCI was introduced to Afghanistan in 2003, with implementation across the country as part of the BPHS. IMCI includes case management of acute respiratory infection, diarrhoea, ear problems, malaria and other febrile illnesses, measles, malnutrition, and anaemia and provides oral drugs and immunisations [84]. Challenges to ensuring the high quality of IMCI services have been documented and primarily relate to the availability of doctors, the receipt of standard IMCI training for health workers, large patient volumes, poor supervision and limited availability of clinical guidelines $[85,86]$. The IMAM is the main malnutrition prevention and treatment strategy. Initially the management of acute malnutrition started as an emergency programme at the community level, introduced as CMAM in 2010. However, to address nutrition prevention and treatment needs comprehensively, the MoPH decided to scale up the management of acute malnutrition through the BPHS and EPHS and shift the focus from emergency services to development and sustainable programming, predominantly at the facility level [33]. Four studies assessed CMAM [70], IMAM [77, 78], and the delivery of nutrition services through the BPHS and EPHS [72]. Across these studies, major constraints to preventing and treating malnutrition at facility and community levels included low nutrition service coverage, shortage of staff, poorly trained staff, and poor supervision and monitoring. Venkataramani et al. [77] found that children who present with IMCI-related complaints (fever, diarrhoea or cough) influence a provider's adherence to the IMCI screening protocol, meaning that children with non-IMCI complaints may be at risk of not being screened for critical but less acutely symptomatic IMCI conditions such as anaemia and malnutrition. Mansoor et al. [78] also found that provider adherence to IMCI algorithms varied and, on average, 5.4 of 10 main assessment tasks were performed during examination of a child. Overall, the nutrition component of the BPHS and EPHS was found to be under-staffed and under-resourced, with optimal nutrition services not delivered [72]. Morikawa et al. [68] evaluated a programme of an inpatient motherchild feeding centre with psychosocial bonding support and supplementary feeding for both mother and child, and growth monitoring for up to 6 months after discharge. They observed significant and continuous improvements in both weight and height during the 6-month follow-up but did not evaluate the impact of an integrated inpatient feeding programme focused on psychosocial bonding on child development and nutrition over the longer term. Mayhew et al. [71] observed the potential impacts of a community growth monitoring programme to aid caretakers in identifying children who had not gained adequate weight and to promote optimal feeding practices for 'catch-up' growth. They recommended a long-term evaluation of the programme using matched pairs of children; however, to date, such a study has not been conducted though similar programmes continue to be implemented. 
Delivery or modelling of a package of community and facility-based interventions, including multisectoral approaches

Packages of community and facility-based nutritionrelated interventions implemented in Afghanistan often included agriculture and livelihoods programmes designed with a nutrition lens, with three large-scale multisector projects to date. In addition to nutrition-specific interventions (e.g. IYCF, CMAM, hygiene awareness), nutritionsensitive interventions in these packages included the provision of seeds, fertiliser and tools for farming [69], kitchen gardens [69, 81], improved latrines [81], dietary diversity awareness $[69,81]$, and small-scale livestock for poultry and dairy production [64, 69, 81]. An assessment of 20 FAO-executed projects [64] found a lack of consistent monitoring and evaluation measures, which impeded accumulation of sufficient data to show meaningful programmatic changes. While other large-scale intervention packages were found to meet their implementation targets [81], these packages of multisectoral interventions were limited in testing combinations of interventions, their delivery approaches and understanding their effectiveness on improving nutrition outcomes. A common thread among multisector programmes was the dependence upon and value of community actors such as volunteer CHWs, Family Health Action groups, community development councils/shuras and WASH groups. These community level actors are crucial for mobilisation efforts and for creating demand for services. Integration of nutrition activities occurred through guidelines developed and used by other agencies, which incorporated nutrition topics into primary education curricula or food processing activities. However, these were implemented directly by a single team comprised of both nutrition and food security specialists working together.

Two studies used the Lives Saved Tool to model the impact of a combination of IYCF interventions, community-based health and nutrition interventions, and WASH on improving child health and nutrition outcomes. Akseer et al. (78) and Higgins-Steele et al. ( [76]) found significant regional variations in coverage and inequalities and that better modelling tools are needed to adequately capture the spectrum of nutrition-sensitive and nutrition-specific interventions and their impacts on child mortality.

\section{Discussion}

This scoping review synthesised information from 18 policies and strategies, 45 data sources and reports, and 20 intervention evaluations relevant to improving nutrition among children in Afghanistan. Key findings from this scoping review include the following: (1) a shift toward multisectoral efforts to address malnutrition at the policy level has started but nutrition-specific and nutrition-sensitive interventions are not yet uniformly delivered or integrated at the community level; (2) political will to improve household nutrition status is reflected in increased government and donor investments in nutrition-sensitive and nutrition-specific programmes through a combination of small- and largescale interventions between 2004 and 2013, but there is limited evidence for interventions that effectively contributed to decreased stunting prevalence; and (3) many data sources capturing nutrition, food security and WASH indicators are available, but efforts are needed to standardise indicator definitions and longitudinal nutrition surveys should be considered to assess change over time and ensure relevance to policy needs.

Aligning with the global momentum for improving nutrition outcomes through a combination of evidencebased nutrition-specific and nutrition-sensitive approaches, Afghanistan has made efforts to prioritise nutrition and food security and, specifically, to address poor child nutrition indicators. However, Afghanistan's progress is undermined by the lack of or weak programmatic efforts to generate demand for nutrition and food interventions, often due to the reliance on volunteers that may not be incentivised to create the necessary level of demand. For example, one nutrition and hygiene promotion programme was pilot-tested among a subset of communities that were a part of a larger unconditional cash transfer project. Evaluators mentioned that participation and interest in the awareness campaigns were high, partly because households had the impression that they might become eligible for the cash transfer programme [79]. Similarly, SMART and SQUEAC assessments found that one of the largest challenges was communities' lack of recognition of malnutrition as a major health concern and their low awareness of malnutrition programmes offered by health facilities [87]. Recognising the need for more innovative forms of demand creation for nutrition information and services, programmes such as the Community-Based Nutrition Package (CBNP), Maternal and Child Health $(\mathrm{MCH})$ handbook, and Community-Led Total Sanitation have recently been introduced and nutrition counsellors have been added as part of BPHS facility staff. The CBNP programme was developed as a comprehensive minimum service package targeting high-impact interventions in the areas of nutrition, WASH and food security for pregnant women and families with children under 24 months of age. It has been implemented in 15 provinces and a recent process evaluation revealed that the CBNP provided adequate quantity and quality of equipment and supplies and refresher training for CHWs [88]. The $\mathrm{MCH}$ handbook was piloted in one district each of Kabul and Nangarhar provinces and distributed to pregnant women or women with children under 24 months of age and promotes all stages of maternal, newborn and child health, including health promotion, hygiene and growth 
monitoring. Based on positive assessment results of the handbook's reach and use, national scale-up over 3 years is planned [89]. Community-Led Total Sanitation has been adopted by the government to mobilise communities to be open defecation-free and to motivate households to improve their traditional latrines; over 1000 communities have remained open defecation-free since 2010 [90]. Progress has also been made towards staffing BPHS facilities with a nutrition counsellor; about $87 \%$ of the planned HFs have been staffed with a nutrition counsellor, of whom $75 \%$ have received the national training package [91].

Ten high impact nutrition-specific interventions across the lifecycle through the first 1000 days were modelled globally to $90 \%$ coverage, which resulted in reducing stunting prevalence by $20 \%$ and wasting prevalence by $60 \%$ [3]. These ten interventions have been implemented in Afghanistan to some degree and include periconceptual folic acid supplementation, maternal balanced energy protein supplementation, maternal calcium supplementation, multiple micronutrient supplementation in pregnancy, promotion of breastfeeding, appropriate complementary feeding, vitamin A administration and preventive zinc supplementation in children 6-59 aged months, and screening for and management of severe acute malnutrition and MAM [3]. Targeting is essential for implementation of high-impact nutrition interventions at scale. Because most interventions in Afghanistan containing knowledge or behaviour change components target mothers and young children (either under 5 years of age or 24 months of age, shown in Table 4), future programmes should consider tailoring messages to men and ensuring greater male engagement as they are the normative decision-makers for healthcare access, household expenditures and foods cultivated within the household. Further, not all pregnant women, mothers and young children require all of the above-mentioned interventions - communities, women and children should be more selectively targeted based on vulnerability and need, allowing for greater cost effectiveness and efficiencies when programming at scale [92]. Multisectoral adaptive management capacity should be strengthened for effective targeting to occur.

Several high level multisectoral policy and strategy documents have been commissioned by the government; government and international partners have supported large-scale nutrition, food security and WASH programmes, though most of these have focused on the health systems supply side and the health sector has worked to strengthen and integrate the nutrition package delivered through its key service delivery platform, the BPHS/EPHS. Given the paucity of evidence in the peer-reviewed literature regarding nutrition interventions in Afghanistan and similar fragile settings, we find current value in grey literature and potentially disagree with excluding this source through systematic reviews or more limited scoping reviews. In countries like Afghanistan, grey literature reports may constitute a substantial amount of contextually relevant data available and accessible to guide policy and programme design. This is likely true for integrated, multisector approaches for improving nutrition, given that evaluation of integrated programmes is complex and methods used are often not appropriately designed [93]. While greater efforts are needed to improve the quality of research in this setting, rapid and well-defined approaches for assessing the whole body of literature - peerreviewed publications and grey literature documents and synthesising evidence for decision-making is needed.

Impacts on nutrition outcomes were not evaluated in any of the included studies due to relatively brief implementation timelines, coverage areas and lack of appropriate study designs. Most evaluations were largely descriptive and focused on programme outputs and targets achieved. Only BPHS and EPHS nutrition services and fortification programmes were designed to reach a national scale but quality, coverage, scope and compliance issues have been reported [72]. All other interventions were implemented in small programme areas, lacking sufficient coverage and scale. Multisector approaches particularly faced challenges of reaching sufficient coverage as they often included a package of interventions across food security, livelihoods and health but were implemented independently of one another. Parallel implementation or improved coordination of multisectoral efforts would be more effective than independent programmes in regions with high malnutrition rates to obtain full-service coverage. Questions remain regarding the most appropriate target group(s), analytic approaches for data from the different delivery models used, suitability of messages, and calculations of costs and benefits. At the policy level, while adequate multisector plans and strategies have been developed, implementation and appropriate coordination mechanisms have delayed their full functionality. Further, at the provincial and field implementation level, coordination structures across ministry sectors are non-existent or remain nascent. The establishment of the AFSeN Secretariat is one high level coordination structure that has been expanded to 12 provincial AFSeN committees. There is currently some uncertainty surrounding continuity and the executive office sponsor, but the provincial AFSeN committees continue policy and programming oversight.

This scoping review shows a breadth of nutrition programme and policy data in Afghanistan. However, measures of nutrition-sensitive and nutrition-specific outcomes, practices and knowledge areas are inconsistent over time and studies are mainly cross-sectional in design. Repeated national cross-sectional surveys remain 
the single source of national nutrition status indicators, such as the most recent AHS 2018, which provides updated information on child nutrition status indicators. The AHS 2018 as compared to the NNS 2013 shows a slight improvement in moderate stunting (36.6\% vs. $40.9 \%)$, severe stunting (17.3\% vs. $20.9 \%$ ), and no improvement in reported exclusive breastfeeding of children under 6 months (57.5\% vs. 58.4\%). However, these variations in study design and key indicators and their definitions preclude meta-analyses. For example, exclusive breastfeeding is a standard IYCF indicator, but there was substantial variation in duration (Table 3) that departs from defined international standards used by WHO and Demographic Health Surveys [34, 36, 39, 41]. Most studies are unable to measure stunting, a main impact indicator, across a shorter time period as it is not sensitive to small changes over time [94] and there is a lack of objective interim measures to assess nutritional status. High levels of stunting are associated with poor socioeconomic conditions; however, improvements in nutrition outcomes over time may not necessarily indicate improved socioeconomic conditions for that specific cohort of children under 5 years of age as they are now school aged [95]. For this reason, longitudinal nutrition studies that monitor linear growth trajectories of children into their early school years would provide a better understanding of intergenerational associations, social differentials and environmental influences [96].

More recent secondary analyses of available data have shown decreasing trends in optimal breastfeeding practices [23] and that socioeconomic inequities are associated with impaired complementary feeding practices [60]. Analysis has also shown positive associations between irrigation, garden plots and dietary diversity, suggesting the need for integration of market strategies, household food production and nutrition education [61]. Current evidence also shows that there are significant district-level geographic disparities in nutrition indicators among children [59]. Improved access to water and sanitation was also a strong predictor of child and maternal nutritional status [59]. Integrated programmes in Afghanistan have not been rigorously evaluated, analysed for combined effects on nutritional status or assessed for optimal implementation strategies. Further research is needed to understand reasons for these declining trends. Implementation evidence is also needed to aid policy and programmes on effective integration of nutrition, food security and WASH. This information should be used for programme planning and targeting beneficiaries.

The all-inclusive approach to large scale programmes allows for piloting and introducing a number of new interventions at once, but testing, refining and identifying what works as a single intervention or as an integrated approach is not often assessed. Small-scale surveys, such as the SMART and SQUEAC surveys, are conducted by implementing partners, but the extent to which data from these surveys have been used to continuously inform and improve their programmes is unknown. Follow-up surveys were not identified nor included in this study.

This scoping review has some limitations. While we did not restrict by time, the exclusion of non-English language documents may have excluded relevant documents. The scoping review methodology allowed for a broad search strategy, which we refined iteratively to maximise eligible source identification. However, some relevant grey literature documents may not have been identified for this review. We were surprised at the minimal inclusion of WASH interventions in eligible reports, considering the long-standing and scaled WASH efforts in Afghanistan. While we aimed to keep our search and inclusion strategy broad, studies were only eligible if relevant nutrition-related indicators, including hygiene promotion, were measured. However, water and sanitation activities may measure outputs of facilities only, which were beyond the scope of our review. We did not assess the quality of the studies included in this review. Evidence for the effectiveness of the evaluated interventions is limited, resulting in studies from Afghanistan being excluded in systematic literature reviews of nutrition intervention efficacy. We attempted to ensure that our inclusion criteria were broad for this scoping review, but only 12 peer-reviewed studies and 8 evaluations from the grey literature were eligible for inclusion. It is notable that 4 grey literature reports assessing nutritionrelated programmes were not included because they were not fully conducted by a third party [97-100]. Donors and implementing partners should ensure that programme evaluations are planned from the beginning with adequate funds allocated.

\section{Conclusion}

The government of Afghanistan, international donors and implementing partners have made important investments in introducing nutrition-specific and nutritionsensitive services across the country. Progress in addressing malnutrition should be accelerated with a focus on integrated multisectoral action.

\section{Supplementary information}

Supplementary information accompanies this paper at https://doi.org/10. 1186/s12961-020-00569-x.

\section{Additional file 1.}

\section{Abbreviations}

ADHS: Afghanistan Demographic and Health Survey; AFSeN: Afghanistan Food Security and Nutrition Agenda; AHS: Afghanistan Health Survey; 
ALCS: Afghanistan Living Conditions Survey; ANDS: Afghanistan National Development Strategy; BPHS: basic package of health services; CBNP: community-based nutrition package; CHWs: community health workers; CMAM: community-based management of acute malnutrition; EBF: exclusive breastfeeding; EPHS: essential package of hospital services; IMAM: integrated management of acute malnutrition; IMCl: integrated management of childhood illness; IYCF: infant and young child feeding; MAIL: Ministry of Agriculture, Irrigation, and Livestock; MAM: moderate acute malnutrition; MICS: Multiple Indicator Cluster Survey; MoPH: Ministry of Public Health; MUAC: Mid-Upper Arm Circumference; NGO: non-governmental organisation; NNS: National Nutrition Survey; NRVA: National Risk and Vulnerability Assessment; SDG: Sustainable Development Goal; SMART: Standardized Monitoring and Assessment of Relief and Transitions; SQUEAC: Semi-Quantitative Evaluation of Access and Coverage; WASH: Water Sanitation and Hygiene

\section{Acknowledgements}

This manuscript is made possible by the support of the American People through the United States Agency for International Development (USAID) The contents of this manuscript are the sole responsibility of Family Health International (FHI 360) and do not necessarily reflect the views of USAID or the United States Government. Specifically, this work was supported through the USAID Integrated Hygiene, Sanitation, and Nutrition contract [AID-306-C16-00007]. We thank key informants for their time and input, stakeholders for contributing requested documents, and FHI 360 internal reviewers for critical comments to improve the quality of this manuscript. We thank Silk Route Training and Research Organization for their contribution to the desk review data collection.

\section{Authors' contributions}

CK contributed to study conception and design, collected and analysed data, and drafted the manuscript. GFM contributed to study conception, data extraction and interpretation, and critical review. MOM contributed to data extraction and critical review. PMP, MJA and MHL contributed to critical review. CT contributed to study conception and design, data extraction and interpretation, and critically revised the draft. All approved the final version submitted.

\section{Funding}

This manuscript is made possible by the support of the American People through the United States Agency for International Development (USAID). The contents of this manuscript are the sole responsibility of Family Health International (FHI 360) and do not necessarily reflect the views of USAID or the United States Government. Specifically, this work was supported through the USAID Integrated Hygiene, Sanitation, and Nutrition contract (AID-306-C16-00007).

\section{Availability of data and materials}

Not applicable. All data reported are publicly available in studies included in this scoping review.

\section{Ethics approval and consent to participate}

Not applicable.

\section{Consent for publication}

Not applicable.

\section{Competing interests}

None declared.

\section{Author details}

${ }^{1}$ Department of Health Policy and Management, Gillings School of Global Public Health, University of North Carolina at Chapel Hill, Chapel Hill, NC, United States of America. ${ }^{2} \mathrm{FHI} 360 /$ Integrated Hygiene, Sanitation, and Nutrition (IHSAN) project, Kabul, Afghanistan. ${ }^{3}$ Public Nutrition Directorate, Ministry of Public Health, Islamic Republic of Afghanistan, Kabul, Afghanistan. ${ }^{4}$ Rural Water Supply and Irrigation Programme (RuWATSIP) Department, Ministry of Rural Rehabilitation and Development (MRRD), Islamic Republic of Afghanistan, Kabul, Afghanistan. ${ }^{5}$ Division of Reproductive, Maternal, Newborn, and Child Health, Global Health, Population and Nutrition Department, Durham, North, Carolina, United States of America.
Received: 10 July 2019 Accepted: 5 May 2020

Published online: 11 June 2020

\section{References}

1. International Food Policy Research Institute. Global Nutrition Report 2016: From Promise to Impact: Ending Malnutrition by 2030. Washington, DC; 2016

2. Levitt E, Kostermans K, Laviolette L, Mbuya N. Malnutrition in Afghanistan: Scale, Scope, Causes, and Potential Response. Washington, DC: The World Bank Institute; 2010.

3. Maternal and Child Nutrition Study Group. Executive Summary of The Lancet Maternal and Child Nutrition Series. Lancet. 2013;5(1):1-11.

4. United Nations. Sustainable Development Knowledge Platform. 2014. https://sustainabledevelopment.un.org/?page=viewandnr=164andtype=230. Accessed 11 Sep 2017

5. Black RE, Victora CG, Walker SP, Bhutta ZA, Christian P, de Onis M, et al. Maternal and child undernutrition and overweight in low-income and middle-income countries. Lancet. 2013;382(9890):427-51.

6. Taylor SAJ, Perez-Ferrer C, Griffiths A, Brunner E. Scaling up nutrition in fragile and conflict-affected states: The pivotal role of governance. Soc Sci Med. 2014;126C:119-27.

7. IFPRI. 2018 Global Hunger Index. 2018. https://www.globalhungerindex.org/ pdf/en/2018.pdf. Accessed 30 Mar 2019.

8. World Bank. Harmonized List of Fragile Situations FY19. Washington, DC; 2019. http://www.worldbank.org/en/topic/fragilityconflictviolence/brief/ harmonized-list-of-fragile-situations. Accessed 3 Jun 2020. .

9. Seal A. Mapping nutrition and health data in conflict-affected countries. Lancet Glob Health. 2018;6(4):e365-6. https://doi.org/10.1016/S2214109X(18)30064-0.

10. Kinyoki DK, Moloney GM, Uthman OA, Kandala N-B, Odundo EO, Noor AM, et al. Conflict in Somalia: impact on child undernutrition. BMJ Glob Health. 2017;2(2):e000262

11. Torlesse H, Aguayo VM. Aiming higher for maternal and child nutrition in South Asia. Matern Child Nutr. 2018;14:e12739 http://doi.wiley.com/10.1111/ mcn.12739.

12. Horton R. Maternal and child undernutrition: an urgent opportunity. Lancet 2008;371(9608):179.

13. Centers for Disease Control and Prevention. Global Diarrhea Burden. https://www.cdc.gov/healthywater/global/diarrhea-burden.html. Accessed 28 Sep 2017.

14. GBD 2015 Mortality and Causes of Death Collaborators. Global, regional, and national life expectancy, all-cause mortality, and cause-specific mortality for 249 causes of death, 1980-2015: a systematic analysis for the Global Burden of Disease Study 2015. Lancet. 2016;388:1459-544.

15. Centers for Disease Control and Prevention. Diarrhea: Common IIIness, Global Killer. 2016. https://www.cdc.gov/healthywater/pdf/global/programs/ globaldiarrhea_asia_508c.pdf. Accessed 3 Jun 2020.

16. Bhutta ZA, Ahmed T, Black RE, Cousens S, Dewey K, Giugliani E, et al. What works? Interventions for maternal and child undernutrition and survival. Lancet. 2008;371(9610):417-40.

17. IFPRI. Why Sanitation Matters for Nutrition. 2015. http://ebrary.ifpri.org/ digital/collection/p15738coll2/id/129074. Accessed 3 Jun 2020.

18. Humphrey JH, Mbuya MNN, Ntozini R, Moulton LH, Stoltzfus RJ, Tavengwa $\mathrm{NV}$, et al. Independent and combined effects of improved water, sanitation, and hygiene, and improved complementary feeding, on child stunting and anaemia in rural Zimbabwe: a cluster-randomised trial. Lancet Glob Health. 2018;7(1):e132-47

19. Coffey D, Spears D. Implications of WASH Benefits trials for water and sanitation. Lancet Glob Health. 2018;6(6):e615. https://doi.org/10.1016/ S2214-109X(18)30229-8.

20. Njuguna J. Effect of eliminating open defecation on diarrhoeal morbidity: An ecological study of Nyando and Nambale sub-counties, Kenya. BMC Public Health. 2016;16:712. https://doi.org/10.1186/s12889-016-3421-2.

21. Fund for Peace. 2018 Fragile States Index. Washington, DC; 2018. https:// fragilestatesindex.org/2018/04/24/fragile-states-index-2018-annual-report/. Accessed 3 Jun 2020.

22. Hunger and Nutrition Commitment Index (HANCI). 2019. http://www. hancindex.org/hanci/. Accessed 30 Mar 2019.

23. Benedict RK, Craig HC, Torlesse H, Stoltzfus RJ. Trends and predictors of optimal breastfeeding among children 0-23 months, South Asia: analysis of national survey data. Matern Child Nutr. 2018;14(S4)):e12697 http://doi.wiley. com/10.1111/mcn.12697. 
24. Goudet S, Murira Z, Torlesse H, Hatchard J, Busch-Hallen J. Effectiveness of programme approaches to improve the coverage of maternal nutrition interventions in South Asia. Matern Child Nutr. 2018;14:e12699.

25. Arksey H, O'Malley L. Scoping studies: towards a methodological framework. Int J Soc Res Methodol. 2005;8(1):19-32.

26. Levac D, Colquhoun H, O'Brien KK. Scoping studies: advancing the methodology. Implement Sci. 2010;5:69.

27. Miyake S, Speakman EM, Currie S, Howard N. Community midwifery initiatives in fragile and conflict-affected countries: a scoping review of approaches from recruitment to retention. Health Policy Plann. 2017;32(1):21-33.

28. Gillespie SR. Strengthening Capacity to Improve Nutrition. Washington, DC: IFPRI, FCND Discussion Paper; 2001. https://www.ifpri.org/publication/ strengthening-capacity-improve-nutrition-0.

29. Ministry of Agriculture Irrigation and Livestock (MAIL) Afghanistan. Food Security and Nutrition (FSN) Strategy. 2015.

30. Ministry of Public Health (MoPH) Afghanistan. National Hygiene Promotion Strategy 2015-2020. 2015.

31. Ministry of Education (MOE), Ministry of Public Health (MoPH), Ministry of Education (MOE). National Rural Water, Sanitation And Hygiene (WASH) Policy 2016-2020. 2016.

32. AFSeN-Technical Secretariat. Afghanistan Food Security and Nutrition Agenda Food Security and Nutrition Public Awareness and Advocacy Framework and Plan 2018-2020. Kabul: Government of the Islamic Republic of Afghanistan. 2018.

33. Ministry of Public Health Afghanistan. Integrated Guidelines for the Management of Acute Malnutrition. 2014.

34. Ministry of Public Health Afghanistan, UNICEF. National Nutrition Survey Afghanistan (2013). 2013.

35. Government of the Islamic Republic of Afghanistan. Ministry of Public Health Afghanistan. 2004 Afghanistan National Nutrition Survey. Kabul; 2004. https://scalingupnutrition.org/wp-content/uploads/2018/10/AFSeN-AFood-Security-and-Nutrtion-Public-Awareness-and-Advocacy-Plan.pdf. Accessed 3 Jun 2020.

36. Central Statistics Organization, Ministry of Public Health Afghanistan, The DHS Program, ICF International. Afghanistan Demographic and Health Survey 2015. 2017. https://dhsprogram.com/pubs/pdf/FR323/FR323.pdf. Accessed 3 Jun 2020.

37. World Health Organization. Guideline: Fortification of Food-Grade Salt with lodine for the Prevention and Control of lodine Deficiency Disorders. Geneva: WHO; 2014

38. Central Statistics Organization Afghanistan. Afghanistan Living Conditions Survey 2013-2014: National Risk and Vulnerability Assessment. 2016. http://eeas.europa.eu/delegations/ukraine/projects/list_ of_projects/projects_en.htm.

39. Ministry of Public Health Afghanistan. 2004 Afghanistan National Nutrition Survey. 2004.

40. Central Statistics Organization (CSO). Afghanistan Living Conditions Survey 2016-17. Kabul; 2018. https://reliefweb.int/report/afghanistan/afghanistanliving-conditions-survey-2016-17. Accessed 3 Jun 2020.

41. The Royal Tropical Institute and Silk Route Training and Research Organization. Afghanistan Health Survey 2015: Final Report. 2016. https:// www.kit.nl/wp-content/uploads/2018/10/08-23-2016-Afghanistan-HealthSurvey-2015-Final-Report.pdf. Accessed 3 Jun 2020.

42. Central Statistics Organization, Ministry of Public Health Afghanistan, The DHS Program, ICF International. Afghanistan Demographic Health Survey 2015: Key Indicators Report. Kabul, Afghanistan; Rockville, MA; 2016.

43. Habib BB. Nutrition and Mortality SMART Survey Final Report: Jawzjan Province, Afghanistan; 2017.

44. Habib BB. Nutrition and Mortality SMART Survey Final Reprot: Farah Province, Afghanistan; 2017.

45. Samim S, Shagiwal Z, Habib BB. Nutrition and Mortality SMART Survey Final Report: Kapisa province, Afghanistan; 2016.

46. Kimanzi S. Semi Quantitative Evaluation of Access and Coverage (SQUEAC) report: Herat Province. Herat: Action Contre la Faim (ACF). 2016.

47. Breitinger JF, Rahim S. Semi-Quantitative Evaluation of Access and Coverage (SQUEAC). Kunar: Action Contre la Faim (ACF). 2015.

48. Ministry of Public Health Afghanistan. Afghanistan National Nutrition Surveillance System Bulletin. 2017

49. Dufour C, Borrel A. Towards a public nutrition response in Afghanistan: Evolutions in nutritional assessment and response. In: Sutton J, Pain A, editors. Reconstructing Agriculture in Afghanistan; 2007.
50. Cheung E, Mutahar R, Assefa F, Ververs MT, Nasiri S, Borrel A, et al. An epidemic of scurvy in Afghanistan: Assessment and response. Food Nutr Bull. 2003;24(3):247-55.

51. Singh M, Nassiri DM, Saidali A, Arya LS. Infant Feeding Practices in Kabul. Indian Pediatr. 1983;20:733-8.

52. Survey S, Report F. Anthropometric and Mortality SMART Survey Final Report: Kandahar Province, Afghanistan; 2016.

53. Newbrander W, Natiq K, Shahim S, Hamid N, Skena NB. Barriers to appropriate care for mothers and infants during the perinatal period in rura Afghanistan: a qualitative assessment. Glob Public Health. 2014;9(Suppl. 1): 93-109. https://doi.org/10.1080/17441692.2013.827735.

54. Benedict RK, Craig HC, Torlesse H, Stoltzfus RJ. Effectiveness of programmes and interventions to support optimal breastfeeding among children 0-23 months, South Asia: a scoping review. Matern Child Nutr. 2018;14:e12697 http://doi.wiley.com/10.1111/mcn.12697.

55. ACF International. Nutrition Anthropometric and Mortality Survey: Province of Ghor. 2014.

56. Hall S. A study of Poverty, Food Security and Resilience in Afghan Cities. 2014. https://www.clovekvtisni.cz/media/publications/729/file/1433502328pin-drc-afghanistan-2014-a-study-of-poverty-food-security-and-resilience. pdf. Accessed 3 Jun 2020.

57. Lautze. Food Insecurity in Afghanistan 1999-2002. 2002.

58. Levitt EJ. Pursuing Food System Approaches to Promote Food Security and Reduce Malnutrition in Afghanistan; 2006.

59. Akseer N, Bhatti Z, Mashal T, Soofi S, Moineddin R, Black RE, et al. Geospatial inequalities and determinants of nutritional status among women and children in Afghanistan: an observational study. Lancet Glob Health. 2018; 6(4):e447-59. https://doi.org/10.1016/S2214-109X(18)30025-1.

60. Na M, Aguayo VM, Arimond M, Mustaphi P, Stewart CP. Predictors of complementary feeding practices in Afghanistan: analysis of the 2015 Demographic and Health Survey. Matern Child Nutr. 2018;14:e12696 http:// doi.wiley.com/10.1111/mcn.12696.

61. Kawsary R, Zanello G, Shankar B. The Role of Irrigation in Enabling Dietary Diversity in Afghanistan. 2018. https://www.eldis.org/document/A102160. Accessed 4 Jun 2020.

62. Grant K, Stone T. Maternal comprehension of a home-based growth chart and its effect on growth. J Trop Pediatr. 1986;32(5):255-7.

63. Kim G, Griffin S, Nadem H, Aria J, Lawry L. Evaluation of an interactive electronic health education tool in rural Afghanistan. Prehosp Disaster Med. 2008;23(3):218-26.

64. Grunewald M, Hahn H, Rafiq H. Report on Tri-partite Evaluation of FAO's German BTF Project GCP/AFG/039/GER Supporting Household Food Security, Nutrition and Livelihoods in Afghanistan; 2008.

65. Manaseki-Holland S, Qader G, Isaq Masher M, Bruce J, Zulf Mughal M, Chandramohan $D$, et al. Effects of vitamin D supplementation to children diagnosed with pneumonia in Kabul: A randomised controlled trial. Trop Med Int Health. 2010:15(10):1148-55.

66. Manaseki-Holland S, Maroof Z, Bruce J, Mughal MZ, Masher MI, Bhutta ZA, et al. Effect on the incidence of pneumonia of vitamin D supplementation by quarterly bolus dose to infants in Kabul: a randomised controlled superiority trial. Lancet. 2012:379(9824):1419-27.

67. Aluisio AR, Maroof Z, Chandramohan D, Bruce J, Mughal MZ, Bhutta Z, et al. Vitamin D3 supplementation and childhood diarrhea: a randomized controlled trial. Pediatrics. 2013;132(4):e832-40.

68. Morikawa M, Polanc A, Becker S. Continuous weight and height gain among at-risk children discharged from a supplementary feeding center in Kabul, Afghanistan. Infant Child Adolesc Nutr. 2013;5(2):97-9.

69. Munroe S. Final Evaluation of Children, Nutrition, and Food Security in Afghanistan; 2013.

70. Ahmed T, Hossain M, Mahfuz M, Choudhury N, Hossain MM, Bhandari N, et al. Severe acute malnutrition in Asia. Food Nutr Bull. 2014;35(2):14-26.

71. Mayhew M, Ickx P, Stanekzai H, Mashal T, Newbrander W. Improving nutrition in Afghanistan through a community-based growth monitoring and promotion programme: a pre-post evaluation in five districts. Glob Public Health. 2014;9(Suppl. 1):S58-75

72. Nasrat Q. Assessment of nutrition interventions in BPHS and EPHS: Final Report; 2014.

73. The World Bank Group. Nutrition Information with the Pilot Cash Transfer Program. Kabul; 2014.

74. Akseer N, Bhatti Z, Rizvi A, Salehi AS, Mashal T, Bhutta ZA. Coverage and inequalities in maternal and child health interventions in Afghanistan. BMC 
Public Health. 2016;16(Suppl. 2):797. https://doi.org/10.1186/s12889-0163406-1.

75. IS Consultancy Services and Save the Children. Improving Communitybased Maternal and Newborn Care in Afghanistan: Operational Research Findings from a Household Survey in Two Provinces. 2016.

76. Higgins-Steele A, Yousufi K, Sultana S, Ali AS, Varkey S. Ending preventable child deaths from pneumonia and diarrhoea in Afghanistan: an analysis of intervention coverage scenarios using the Lives Saved Tool. J Trop Med. 2017;2017:3120854.

77. Venkataramani M, Edward A, Ickx P, Younusi M, Alawi SAS, Peters DH. Are children presenting with non-IMCl complaints at greater risk for suboptimal screening? An analysis of outpatient visits in Afghanistan. Int J Qual Health Care. 2017;29(5):662-8.

78. Mansoor GF, Chikvaidze P, Varkey S, Higgins-Steele A, Safi N, Mubasher A, et al. Quality of child healthcare at primary healthcare facilities: a national assessment of the Integrated Management of Childhood IIInesses in Afghanistan. Int J Qual Health Care J Int Soc Qual Health Care. 2017;29(1): $55-62$.

79. The World Bank Group. Nutrition Information with the Pilot Cash Transfer Program. Kabul: World Bank; 2014. .

80. Global Alliance for Improved Nutrition. Fortification Assessment Coverage Toolkit (FACT) Survey in Afghanistan, 2017. Geneva: GAIN; 2017.

81. Pedersen S, Ayan M, Sibghatullah D, Erfani S, Noorzad S. Final Evaluation of the Maternal and Under-Five Nutrition and Child Health Project. Kabul: Checchi and Company Consulting, Inc.; 2016.

82. World Bank. Increasing the Nutritional Impact of the National Horticulture and Livestock Productivity Project. 2014.

83. World Health Organization. Integrated Management of Childhood IIIness (IMCI). 2019. https://www.who.int/maternal_child_adolescent/topics/child/ imci/en/. Accessed 4 Jun 2020

84. MOPH Afghanistan. A Basic Package of Health Services for Afghanistan 2010/1389. 2010. http://www.moph.gov.af/en/index.php?id=23.

85. Mayhew M, Ickx P, Newbrander W, Stanekzai H, Alawi SA. Long and short Integrated Management of Childhood Illness (IMCI) training courses in Afghanistan: a cross-sectional cohort comparison of post-course knowledge and performance. 2015;4(3):143-52. https://doi.org/10.15171/ijhpm.2015.17.

86. Edward A, Kumar B, Niayesh H, Naeem AJAN, Burnham G, Peters DH. The association of health workforce capacity and quality of pediatric care in Afghanistan. Int J Qual Health Care. 2012;24(6):578-86.

87. Coghlan B, Chan G, Power R. Participatory research: Children of Uruzgan Program. Melbourne: Burnet Institute; 2014.

88. Koichiro W. Community based nutrition package process evaluation in Afghanistan 2018-2019. Kabul: UNICEF Afghanistan Country Office; 2020.

89. Saeedzai SA, Sadaat I, Anwari Z, Hemat S, Hadad S, Osaki K, et al. Homebased records for poor mothers and children in Afghanistan, a cross sectional population based study. BMC Public Health. 2019;19:766.

90. Ministry of Rural Rehabilitation and Development (MRRD). Implementation manual: Community led total sanitation (CLTS). 2016. https://www. communityledtotalsanitation.org/sites/communityledtotalsanitation.org/files/ CLTS_Implementation_Manual_AFghanistan.pdf. Accessed 3 Jun 2020.

91. Sayara Research. Third Party Monitoring (TPM) of Nutrition Counsellors' Performance in 18 Provinces: Assessment Report. Kabul: Sayara Research; 2018.

92. Victora CG, Barros FC, Assunção MC, Restrepo-Méndez MC, Matijasevich A, Martorell R. Scaling up maternal nutrition programs to improve birth outcomes: a review of implementation issues. Food Nutr Bull. 2012; 33(Suppl. 2):6-26.

93. FHI 360. Guidance for Evaluating Integrated Global Development Programs. Durham: FHI 360; 2016. https://www.fhi360.org/resource/guidanceevaluating-integrated-global-development-programs. Accessed 3 Jun 2020.

94. Stunting Prevalence in Children Under 5. 2016. https://www. righttofoodandnutrition.org/stunting. Accessed 18 Mar 2019.

95. World Health Organization. Global Database on Child Growth and Malnutrition . Global Database on Child Growth and Malnutrition. 1997. http://www.who.int/nutgrowthdb/about/introduction/en/index2.html. Accessed 25 Mar 2015

96. Ekholm Selling K, Svefors P, Rahman A, Khan Al, Ekström E-C, Lindström E, et al. Stunted at 10 Years. Linear growth trajectories and stunting from birth to pre-adolescence in a rural Bangladeshi cohort. PLoS One. 2016;11(3): e0149700.
97. Timpl G, Knox S. Improving Nutrition for Mothers, Newborns and Children in Afghanistan: Endline Evaluation Report; 2017.

98. Kittle B, Sadruddin S, IHSAN T. Provincial Strengthening in Northern Afghanistan: Capacity Building and Innovation to Support the Basic Package of Health Services and Sustainably Improve Access, Quality and Use of Essential MCH Services throughout Jawzjan Province; 2008.

99. The Home Fortification Technical Advisory Group (2014) Home Fortification with Micronutrient Powders (MNP), Sight and Life. Basel. https://issuu.com/ sight_and_life/docs/mnp_supplement_to_27_2_2013. Accessed 3 Jun 2020.

100. World Vision Inc. Despite Hostile Terrain and Social Barriers: An Evaluation of the Better Health for Afghan Mothers and Children Project. 2013. https:// www.globalwaters.org/resources/assets/despite-hostile-terrain-and-socialbarriers-evaluation-better-health-afghan-mothers. Accessed 4 Jun 2020.

\section{Publisher's Note}

Springer Nature remains neutral with regard to jurisdictional claims in published maps and institutional affiliations.
Ready to submit your research? Choose BMC and benefit from:

- fast, convenient online submission

- thorough peer review by experienced researchers in your field

- rapid publication on acceptance

- support for research data, including large and complex data types

- gold Open Access which fosters wider collaboration and increased citations

- maximum visibility for your research: over $100 \mathrm{M}$ website views per year

At BMC, research is always in progress.

Learn more biomedcentral.com/submissions 